\title{
Individual Differences in Behavioral Estimates of Cochlear Nonlinearities
}

\author{
Gayla L. Poling $^{1,2}$, Amy R. Horwitz ${ }^{1}$, Jayne B. Ahlstrom ${ }^{1}$, and Judy R. Dubno ${ }^{1}$ \\ ${ }^{1}$ Department of Otolaryngology-Head and Neck Surgery, Medical University of South Carolina, 135 Rutledge Avenue, \\ MSC 550, Charleston, SC 29425, USA \\ ${ }^{2}$ Roxelyn and Richard Pepper Department of Communication Sciences and Disorders, Northwestern University, Evanston, IL \\ 60208, USA
}

Received: 8 March 2011; Accepted: 29 August 2011; Online publication: 22 September 2011

\begin{abstract}
Psychophysical methods provide a mechanism to infer the characteristics of basilar membrane responses in humans that cannot be directly measured. Because these behavioral measures are indirect, the interpretation of results depends on several underlying assumptions. Ongoing uncertainty about the suitability of these assumptions and the most appropriate measurement and compression estimation procedures, and unanswered questions regarding the effects of cochlear hearing loss and age on basilar membrane nonlinearities, motivated this experiment. Here, estimates of cochlear nonlinearities using temporal masking curves (TMCs) were obtained in a large sample of adults of various ages whose hearing ranged from normal to moderate cochlear hearing loss (Experiment 1). A wide range of compression slopes was observed, even for subjects with similar ages and thresholds, which warranted further investigation (Experiment 2). Potential sources of variance contributing to these individual differences were explored, including procedural-related factors (test-retest reliability, suitability of the linearreference TMC, probe sensation levels, and parameters of TMC fitting algorithms) and subject-related factors (age and age-related changes in temporal processing, strength of cochlear nonlinearities estimated with distortion-product otoacoustic emissions, estimates of changes in cochlear function from damage to outer hair cells versus inner hair cells). Subject age did not
\end{abstract}

Correspondence to: Judy R. Dubno - Department of OtolaryngologyHead and Neck Surgery - Medical University of South Carolina . 135 Rutledge Avenue, MSC 550, Charleston, SC 29425, USA. Telephone: +1-843-792-7978; fax: +1-843-792-7736; dubnojr@musc.edu contribute significantly to TMC or compression slopes, and TMC slopes did not vary significantly with threshold. Test-retest reliability of TMCs suggested that TMC masker levels and the general shapes of TMCs did not change in a systematic way when re-measured many weeks later. Although the strength of compression decreased slightly with increasing hearing loss, the magnitude of individual differences in compression estimates makes it difficult to determine the effects of hearing loss and cochlear damage on basilar membrane nonlinearities in humans.

Keywords: basilar membrane responses, compression, human, temporal masking curves, hearing loss, aging

\section{INTRODUCTION}

The ability to perceive sounds over a wide range of sound pressure levels has been attributed to the normal functioning of outer hair cells and basilar membrane compression. Physiological evidence indicates that the healthy basilar membrane is sensitive to very low-level sounds and that the response of the basilar membrane grows compressively as sound level increases (see Robles and Ruggero 2001 for review). Although the basilar membrane response is highly compressive close to the characteristic frequency $(\mathrm{CF})$, the response to signals at frequencies well below the CF (at least for high CFs) is more linear regardless of input level (Rhode and Cooper 1996). Damage to outer hair cells results in elevated thresholds, narrower dynamic ranges, less compressive response 
growth, and poorer tuning (e.g., Ruggero et al. 1997). Several behavioral methods have been proposed to estimate cochlear nonlinearities in humans, including growth-of-masking functions (Oxenham and Plack 1997), additivity of masking (Plack et al. 2006), and temporal masking curves (TMC; Nelson et al. 2001). These methods provide a way to infer the characteristics of the basilar membrane response in humans that cannot be directly measured (see Oxenham and Bacon 2003 for review). Because these measures are indirect, the interpretation of results depends on several underlying assumptions. Investigations of the suitability of these assumptions and, in turn, the most appropriate measurement and compression estimation procedures are ongoing (e.g., Wojtczak and Oxenham 2009). Here, the focus is on the TMC method, a commonly used technique, which has been considered among the more accurate methods because it minimizes off-frequency listening.

The TMC method described by Nelson et al. (2001) involves a forward-masking task whereby the masker level required to just mask a fixed, low-level pure-tone probe is measured as a function of the masker-probe interval to produce a temporal masking curve. Limiting the probe to a low level minimizes spread of excitation along the basilar membrane and effects of off-frequency listening (Nelson et al. 2001). Because the probe level is fixed, the masker level needed increases with increasing masker-probe interval, resulting in TMCs that have positive slopes. For an offfrequency masker, which is assumed to be processed linearly, the TMC is assumed to reflect decay of masking. As the masker-probe interval is increased, the masker level required at masked threshold increases to compensate for the time course of decay. For an onfrequency masker, the TMC is assumed to reflect the combined effects of the decay of masking and compression applied to the masker. Therefore, a larger change in masker level would be required to produce a given change in basilar membrane excitation when the response to the masker is compressive. This would be reflected as a steeper on-frequency TMC compared to an off-frequency TMC. Assuming the time course of decay of the masker is identical for all masker frequencies (and levels), the degree of basilar membrane compression can be estimated by comparing the slope of a TMC for an on-frequency masker against the slope of a TMC for a masker that is processed linearly by the basilar membrane (i.e., off-frequency masker or linearreference TMC). Basilar membrane responses can be inferred by plotting the off-frequency masker level against the on-frequency masker level required for each masker-probe interval.

The accuracy of compression estimates obtained with TMCs depends on the selection of a suitable linear reference. Citing physiologic data (e.g., Yates
1990; Ruggero 1992; Rhode and Recio 2000) and behavioral data (e.g., Nelson and Schroder 1997; Oxenham and Plack 1997), Nelson et al. (2001) proposed using an off-frequency masker that is nearly an octave below the probe frequency, assuming it produces a linear response at the probe-frequency place in the cochlea. Subsequently, Lopez-Poveda et al. (2003) proposed using off-frequency maskers measured for higher frequency probes, citing physiological evidence that compression extends to lower frequencies (relative to CF) for apical cochlear sites (Rhode and Cooper 1996). However, this suggestion requires an additional assumption, namely that the rate of decay of forward masking is the same for all probe frequencies. Further, Lopez-Poveda and AlvesPinto (2008) reported that for a $4.0-\mathrm{kHz}$ probe frequency, basilar membrane responses to a $2.2-\mathrm{kHz}$ masker (i.e., 0.55* probe frequency) showed approximately 2:1 compression, whereas responses to a $1.6-\mathrm{kHz}$ masker (i.e., $0.40 *$ probe frequency) reflected linear basilar membrane responses. In addition to the frequency separation between the probe and masker, other important factors may include the absolute levels of the maskers and effects related to the medial olivocochlear reflex (MOCR; e.g., Wojtczak and Oxenham 2010).

In addition to the ongoing uncertainties concerning parameters required for a suitable linear-reference TMC, a more complete understanding of compression estimates obtained from TMCs is further complicated by the limited data obtained from individuals with cochlear hearing loss. Previous studies have included subjects with a range of hearing loss (mild to severe) and a variety of signal and masker frequencies and levels (Nelson et al. 2001; Plack et al. 2004; Rosengard et al. 2005). Plack et al. (2004) examined the impact of hearing loss on the basilar membrane response by comparing behavioral measures obtained from individuals with mild to moderate cochlear hearing loss (12 ears) to those with normal hearing (16 ears). Slopes for both off- and on-frequency TMCs were shallower for hearing-impaired than for normal-hearing subjects. The shapes of inferred basilar membrane input-output responses derived from the on-frequency and off-frequency TMCs suggested a compressive region at high levels and a truncated linear region at lower levels. Plack et al. (2004) interpreted these findings as indicating reduced gain for lower level CF tones, with little change in maximum compression associated with mild-to-moderate hearing loss. Rosengard et al. (2005) investigated compression effects in individuals with normal hearing and hearing loss ranging from mild to severe (five listeners in each group). Subjects with more hearing loss had slopes that were generally similar for the off- and on-frequency TMCs. Consistent with Plack et al. (2004), slopes for both off- and on-frequency TMCs were shallower for 
hearing-impaired than for normal-hearing subjects. With very shallow TMC slopes resulting from restricted ranges of masker levels, derived input-output functions cover a restricted range of input and output levels, thereby providing an incomplete description of the basilar membrane response. In contrast to Plack et al. (2004), Rosengard et al. (2005) found no evidence for a range of levels over which compression was near normal. Stainsby and Moore (2006) obtained TMCs for three ears with moderate sensorineural hearing loss for probe frequencies from 0.5 to $6.0 \mathrm{kHz}$. Most TMCs were well-fit by straight lines that were roughly parallel for each probe frequency tested, resulting in linear response functions consistent with an absence of basilar membrane compression. However, slopes of the TMCs for 0.5 and $1.0 \mathrm{kHz}$ were steeper than for higher frequencies, calling into question the assumption that the rate of decay of masking is the same for all probe frequencies. Most recently, Jepsen and Dau (2011) obtained TMCs for three normal-hearing and 10 hearing-impaired subjects to estimate compression at 1.0 and $4.0 \mathrm{kHz}$ and found that compression slope generally increased with pure-tone thresholds, but noted "remarkable variation" in input-output functions among individuals with similar audiograms.

Given these conflicting findings, the effect of hearing loss on behavioral estimates of cochlear nonlinearities is unclear. It remains to be determined to what extent the variation observed across previous studies reflects true differences in cochlear compression among individuals or measurement factors, such as choice of linear-reference TMC and fitting algorithms for inferred input-output responses. The issue may be further complicated by the contribution of subject age, which has not been explored systematically in sample sizes large enough and age ranges wide enough to permit separating effects of age and hearing loss (Lopez-Poveda and Alves-Pinto 2008). The present study investigated the use of the TMC method to estimate cochlear nonlinearities in a large sample of adults of various ages and with hearing thresholds ranging from normal to moderate hearing loss. TMCs were measured and basilar membrane responses were inferred to explore the impact of hearing loss and age on compression estimates (Experiment 1). Large individual differences observed in TMCs and compression estimates prompted investigation of potential sources of this variance (Experiment 2).

\section{EXPERIMENT 1}

Methods

Subjects. Fifty-one adults, ranging in age from 19 to 87 years (mean age $=55.3$ years; 19 males; 32 females), participated in this experiment. Recruitment efforts focused on obtaining subjects across a continuum of ages and hearing thresholds. All subjects provided written informed consent prior to their participation, which was approved by the Institutional Review Board of the Medical University of South Carolina. For data analysis, subjects were organized into three groups according to the threshold for the probe measured in quiet (described in the next section): (1) low threshold group $(n=24$; mean age $=39.6$ years, range $=$ 19-77); (2) mid threshold group ( $n=11$; mean age $=$ 59.3 years, range $=22-80$ ) ; and $(3)$ high threshold group $(n=16$; mean age $=76.1$ years, range $=65-87)$. Mean audiometric thresholds (in dB HL) for the right (test) ear for each group are shown in Figure 1.

Stimuli and apparatus. TMCs were measured for a $1.0-\mathrm{kHz}$ probe at $10 \mathrm{~dB}$ above the quiet threshold for the probe as a function of the time interval between the masker and the probe, for on-frequency $(1.0 \mathrm{kHz})$ and off-frequency $(0.5 \mathrm{kHz})$ maskers. The $1.0-\mathrm{kHz}$ probe was selected to complement data being collected in a parallel study measuring detection of gaps in noise markers centered at $1.0 \mathrm{kHz}$ (Horwitz et al. 2011). Masker-probe intervals ranged from 0 to $70 \mathrm{~ms}$ in 10-ms steps targeting a minimum of seven masker-probe intervals. For cases where masker levels would have exceeded maximum level restrictions using 10-ms steps, 5-ms steps were used. Probe and masker durations were 20 and $200 \mathrm{~ms}$, respectively, with 10-ms raised-cosine rise and fall ramps.

During data collection for TMCs, the subject was seated inside a double-walled, sound-attenuating booth and registered responses via a button box (TDT RBOX). The probe and maskers were digitally generated with custom Labview software (Labview 8.5,

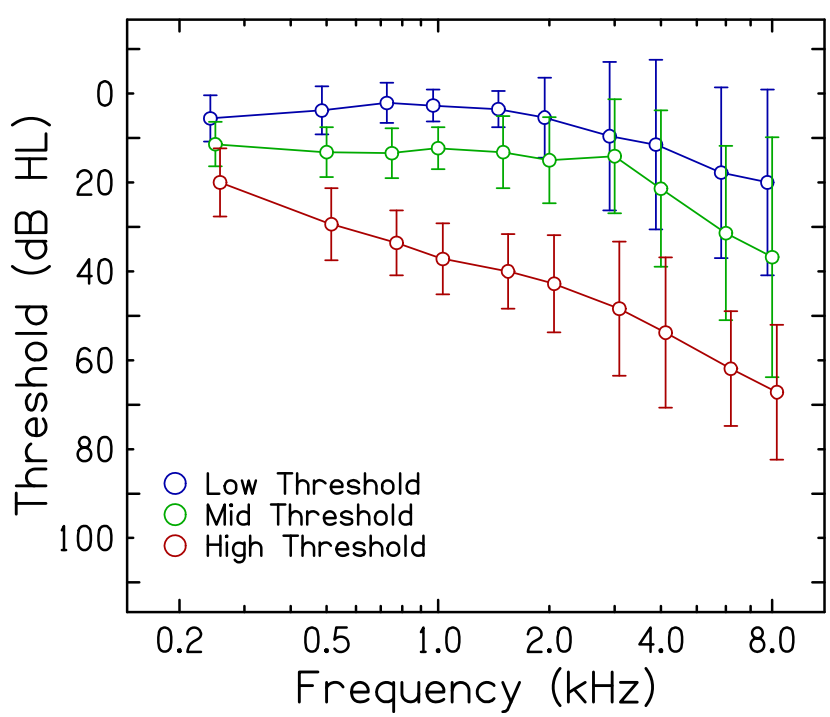

FIG. 1. Mean audiometric thresholds for the right (test) ear for three subject groups, low threshold (b/ue), mid threshold (green), and high threshold (red). Error bars represent \pm 1 standard deviation. For clarity, data points are offset along the abscissa. 
National Instruments) and converted to analog using two channels of a 16-bit digital-to-analog converter (National Instruments, model 6052E) with a sampling rate of $50 \mathrm{kHz}$. The amplitudes of all signals were controlled individually using fixed attenuators (TDT PA4). The probe was added to the masker (TDT SM3) and then passed through a headphone buffer (TDT HB5) for monaural presentation to the test ear through TDH-39 (Telephonics) headphones.

Procedures. A three-interval, three-alternative forced-choice adaptive procedure with feedback was used to measure masker levels and detection thresholds for the probe and maskers. The adaptive procedure converged on the $70.7 \%$ point with a twodown, one-up tracking technique (Levitt 1971). The probe level was fixed at $10 \mathrm{~dB}$ SL and masker level was varied adaptively. The step size of the adaptive track was $4 \mathrm{~dB}$ for the first four reversals and then reduced to $2 \mathrm{~dB}$ for the subsequent eight reversals. A run terminated after 12 reversals, and a threshold estimate was obtained by averaging the masker levels at the last six reversals. The maximum allowable masker level was set at $102 \mathrm{~dB}$ SPL; the run was aborted if the masker level determined by the adaptive track would have exceeded this limit. A threshold measurement was discarded and repeated if the standard deviation of masker levels of the last six reversals exceeded $6 \mathrm{~dB}$ (Lopez-Poveda and Alves-Pinto 2008). Three threshold estimates were obtained and averaged at each masker-probe interval. When the standard deviation of the mean of those three thresholds exceeded $6 \mathrm{~dB}$, a fourth estimate was obtained (this occurred in $17 \%$ of cases) and included in the average (Lopez-Poveda and Alves-Pinto 2008). Therefore, three to four threshold estimates were obtained for each masker-probe interval.

Test order for the masker (on- or off-frequency) and masker-probe intervals ( 0 to $70 \mathrm{~ms}$ ) were selected randomly for each subject. Masker levels were obtained for a complete set of masker-probe intervals, alternating between maskers. Quiet thresholds for the $1.0-\mathrm{kHz}$ probe were measured first using the same adaptive paradigm; when the standard deviation of the means of three threshold estimates exceeded $3 \mathrm{~dB}$, a fourth estimate was obtained (this occurred once) and included in the average.

Each subject practiced the TMC task prior to data collection. Practice started with a longer maskerprobe interval (i.e., 60 or $70 \mathrm{~ms}$ ) and the offfrequency masker to ensure that the probe was relatively easy to hear and that the subject understood the task. These conditions, for which the masker level would most likely exceed the maximum, also determined if a shorter maximum masker-probe interval and 5-ms step sizes were required for that subject. Additional masker-probe intervals were practiced until the subject was familiarized with the task. Thresholds obtained during this practice session were discarded. During each subsequent day of data collection, the first run collected was also discarded.

Fitting procedures for TMCs and inferred input-output responses. Estimating compression from TMCs involves inferring basilar membrane input-output responses by plotting the level of the off-frequency masker against the level of the on-frequency masker for each masker-probe interval. For cases where off-frequency masker levels at longer masker-probe intervals would have exceeded the maximum permissible level, a maximum of one extrapolated point in the off-frequency TMC was included where a corresponding on-frequency data point had been collected. To obtain the extrapolated point, a straight-line fit was used (if the number of measured points in the off-frequency TMC was $\geq 3$ ) or a double exponential function was used (if the number of measured points was $\geq 5$; Johannesen and Lopez-Poveda 2008). The extrapolated thresholds for these two procedures differed by $<5 \mathrm{~dB}$ and, with the limited data available, the straight-line fit could not be reliably differentiated from the nonlinear fit. Therefore, a straight-line fit was used to generate an extrapolated point, when needed. Finally, slopes of off-frequency TMCs were estimated using straight-line fits.

A three-segment linear regression procedure, reported by Yasin and Plack (2003) and Plack et al. (2004), was used to infer the basilar membrane input-output response. The three-segment fit corresponds to the linear-compressed-linear segments typical of these functions and was used to estimate the breakpoints (the transition point from the linear to the compressed segments and a second transition point from the compressed segment to the higher level linear segment) and the slope of the mid-level compressed segment. A custom Matlab program including the fminsearch function was used, whereby the slopes of the lower and upper segments were fixed at 1.0 (linear response). The slope of the mid-level segment and the location of the lower and upper breakpoints joining the three segments were varied by the fitting procedure to satisfy a least-squares regression criterion. At least five points were required for the fitting algorithm (Plack et al. 2004), with at least three of those points falling in the compressed region. The root-mean-square (rms) error between fitted and measured values must be $<5 \mathrm{~dB}$. Using these criteria, compression slope estimates were obtained for 43 of 51 subjects. An estimate of breakpoint was included only if at least one measured point occurred below the estimated breakpoint, which was the case for 27 of 51 subjects.

In addition to the three-segment linear regression, a third-order polynomial fit (Johannesen and LopezPoveda 2008) was used to infer basilar membrane 
input-output responses. Compression slopes estimated by the two procedures were similar and significantly correlated $(N=25 ; r=0.543, p=0.004)$. Because data from more subjects could be fit with the three-segment regression procedure than with third-order polynomials, only responses inferred using three-segment fits are presented here.

\section{Results and discussion}

Compression estimates from inferred input-output responses. Compression slope estimates plotted against threshold for the $1.0-\mathrm{kHz}$ probe are shown in Figure 2 (top). Compression slopes ranged from strongly compressive to expansive, even among subjects with similar thresholds. Specifically, slopes ranged from 0.083 to $1.749 \mathrm{~dB} / \mathrm{dB}$ in the low threshold group, 0.150 to $0.862 \mathrm{~dB} / \mathrm{dB}$ in the mid threshold group, and 0.174 to $1.648 \mathrm{~dB} / \mathrm{dB}$ in the high threshold group. Using an analysis of covariance (ANCOVA) with threshold as a grouping factor and age as a covariate, no significant main effects of threshold group $[F(2,39)=1.90, p=0.163]$ or age $[F(1,39)=0.04$, $p=0.839]$ on compression slope were observed. Removing age from the model in a subsequent ANOVA assessing the effect of threshold group did not affect the results.

As shown in Figure 2, compression slopes increased slightly but significantly with increasing probe threshold ( $r=0.302, p=0.049)$. The slope of the regression indicates that for each 10-dB increase in probe threshold, compression slope increases by $0.088 \mathrm{~dB} / \mathrm{dB}$. Moreover, the modest correlation means that probe threshold accounted for only $9.1 \%$ of the variance in compression slope. These results were further examined using Mplus (Version 5.2) with a path analysis (Loehlin 2004), which uses a different model to determine effects of threshold on compression slope (while controlling for correlations among other variables, including age). Probe threshold was found to account for a nonsignificant $10.8 \%$ of the variance in slope $(p=0.091)$. Taken together, these analyses suggest that the contribution of threshold to compression slope is small and that the magnitude of individual differences makes it difficult to offer a strong conclusion about the relationship between hearing loss and compression slope.

Figure 2 (bottom) shows breakpoints plotted against probe threshold. Breakpoints ranged from 18.7 to $42.7 \mathrm{~dB}$ in the low threshold group, 34.8 to $56.7 \mathrm{~dB}$ in the mid threshold group, and 57.5 to $69.3 \mathrm{~dB}$ in the high threshold group. Using ANCOVA with threshold as a grouping factor and age as a covariate, a significant main effect of threshold group on breakpoint was found $[F(2,23)=25.52, p<0.001]$, but no significant main effect of age $[F(1,23)=1.82, p=$
0.190]. Removing age from the model in a subsequent ANOVA assessing the effect of threshold group did not affect the results. Breakpoints were strongly positively correlated with probe threshold ( $r=0.915$; $p<0.001)$, such that subjects with higher probe thresholds had higher breakpoints. The slope of the regression was nearly linear $(1.1 \mathrm{~dB} / \mathrm{dB})$, indicating that for every $\mathrm{dB}$ increase in probe threshold, breakpoint increases by approximately the same amount $(1.1 \mathrm{~dB})$. Using Mplus with a path analysis, probe threshold was found to uniquely account for $76.6 \%$ of the variance in breakpoints $(p<0.001)$. Breakpoint estimates and the strong association with probe threshold are consistent with previous findings derived from growth-of-masking functions at higher
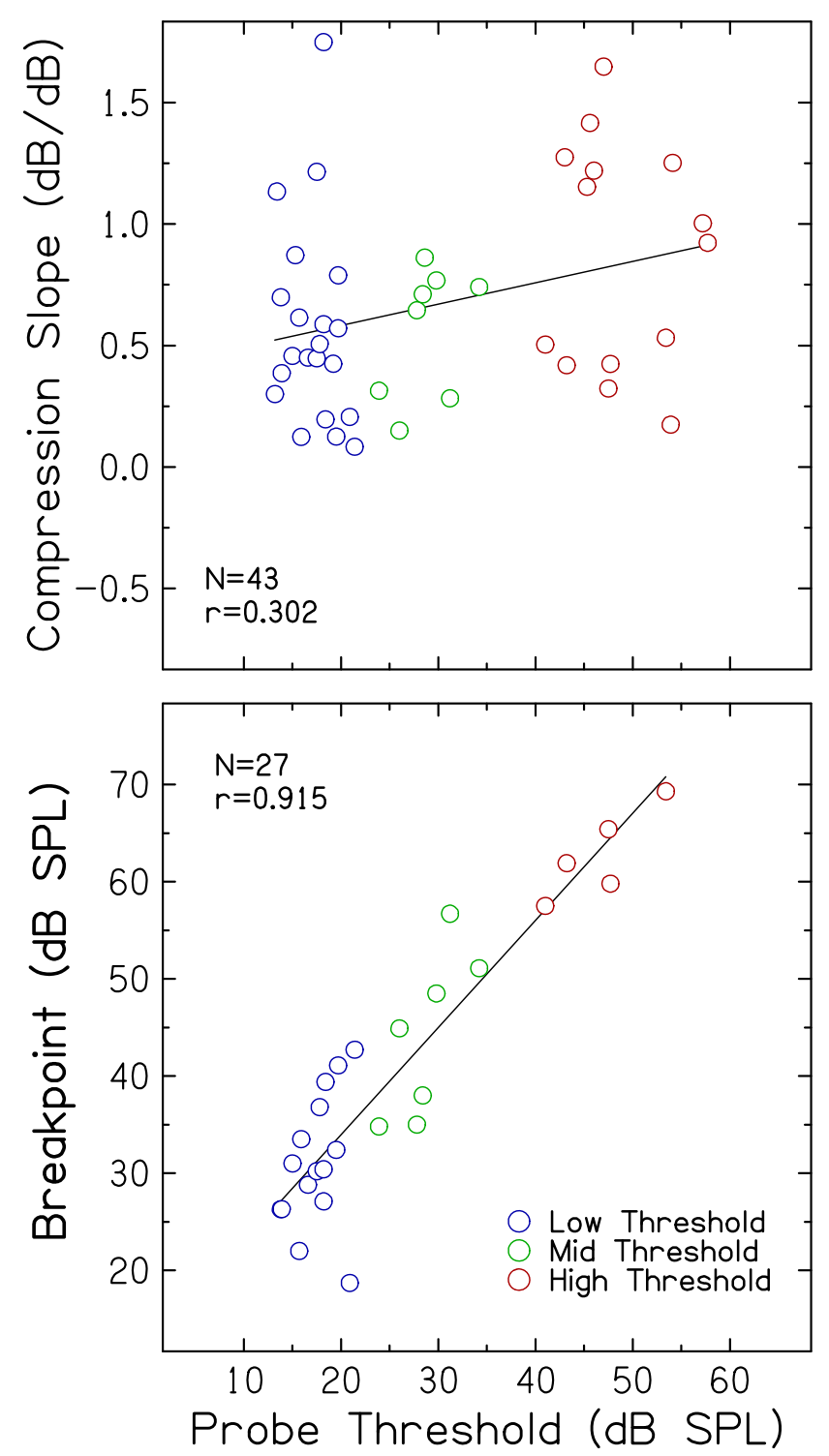

FIG. 2. Top compression slope estimates plotted against threshold for the probe $(1.0 \mathrm{kHz})$. Bottom same as top panel, but for breakpoints of input-output functions. Pearson correlation coefficients and linear regression functions are included in each panel. 
frequencies (Oxenham and Plack 1997; Dubno et al. 2007; Horwitz et al. 2007).

The current results showing a range of compression estimates and a slight increase in compression slope with increasing threshold are generally consistent with previous studies that included hearingimpaired subjects. With fewer subjects, Rosengard et al. (2005) also estimated steeper compression slopes for hearing-impaired than for normal-hearing subjects. Results from Plack et al. (2004) showed a non-significant trend for slightly increasing compression slope with increasing hearing loss. Estimates from some hearing-impaired subjects with shallow slopes were based on line fits with only one or two values within the compressed region or relied on extrapolated off-frequency data. Measuring growth-of-masking functions at 2.0 and $4.0 \mathrm{kHz}$ instead of TMCs, we also reported a range of compression slopes for subjects with similar thresholds (Dubno et al. 2007; Horwitz et al. 2007); fewer subjects with elevated thresholds were included. Although an increasing trend in slopes was seen in some cases, compression slope did not vary significantly with probe threshold. These differences and similarities across studies highlight the uncertainty remaining regarding the nature of the effect of hearing loss on basilar membrane compression in human subjects.

On-frequency and off-frequency TMCs. Analysis of TMCs included a subset of subjects $(N=36)$ for whom masker levels were available for on- and offfrequency maskers for six masker-probe intervals $(0$, $10,20,30,40$, and $50 \mathrm{~ms}$ ). The same pattern of results was obtained when seven masker-probe intervals were included (0-60 ms), with data from fewer subjects available $(N=32)$. Masker levels were examined using a repeated-measures ANCOVA with masker type (onand off-frequency) and masker-probe interval as within-subjects factors, and probe threshold as a covariate. Because age did not show a significant effect when added as a covariate $[F(1,32)=0.29, p=$ 0.592], age was not included in this ANCOVA.

Significant interactions were found between masker type and masker-probe interval $[F(5,170)=6.30 ; p<$ $0.001]$ and among masker type, masker-probe interval, and probe threshold $[F(5,170)=4.14 ; p=0.001]$. The interaction between masker type and masker-probe interval simply reflects the expected differences in masker levels for off- and on-frequency maskers as the duration between the masker and probe increases, which is the basis for the TMC method. The three-way interaction with probe threshold reflects that the interval-dependent differences in masker levels for offand on-frequency maskers change with increases in hearing loss at the probe frequency.

One of the assumptions of the TMC method is that slopes of the off-frequency TMCs depend only on the decay of masking. However, slopes of off-frequency TMCs have been shown in previous studies to become shallower with increasing hearing loss (Plack et al. 2004; Rosengard et al. 2005; Lopez-Poveda et al. 2005). To determine the extent to which slopes of off-frequency TMCs vary with probe threshold in this large dataset, slopes plotted against probe threshold are shown in Figure 3. As noted previously for compression slope estimates, large individual differences in offfrequency TMC slopes were observed for subjects with similar thresholds, even individuals with essentially normal hearing. For example, off-frequency TMC slopes as steep as $0.6-0.8 \mathrm{~dB} / \mathrm{dB}$ were observed for subjects whose probe thresholds ranged from $\sim 10$ to $20 \mathrm{~dB}$ SPL. Although there is a trend for shallower slopes with increasing hearing loss, off-frequency TMC slope did not vary significantly with probe threshold $(r=-0.216 ; p=0.129)$. These results were further examined using Mplus with a path analysis to determine effects of threshold on TMC slope. Probe threshold was found to account for only $0.08 \%$ of the variance in off-frequency TMC slope $(p=0.875)$, which is not consistent with previous findings (e.g., Plack et al. 2004; Lopez-Poveda et al. 2005), perhaps due to the larger sample size and wider and continuous distributions of probe thresholds in the current experiment.

A linear reference can be difficult to obtain for subjects with hearing loss because the required masker levels may exceed maximum limits, especially at higher frequencies where hearing loss is greater. As noted earlier, one factor in the selection of signal frequencies in the current experiment was a parallel study measuring gap detection in noise markers centered at $1.0 \mathrm{kHz}$. As a compromise to meet all

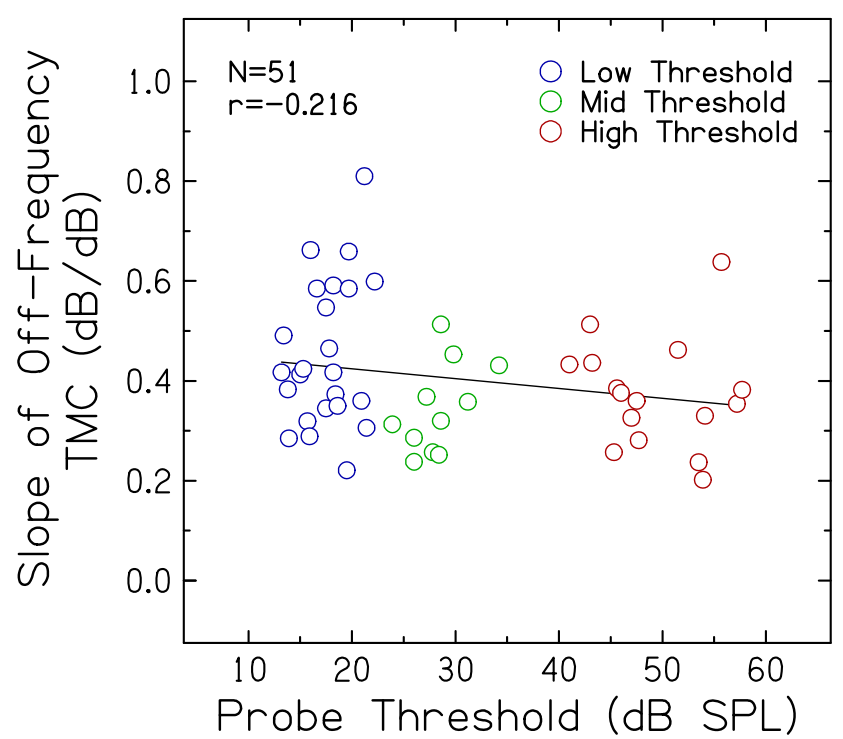

FIG. 3. Slopes of off-frequency TMCs plotted against probe threshold. The Pearson correlation coefficient and linear regression function are included. 
requirements, a $1.0-\mathrm{kHz}$ probe, $1.0-\mathrm{kHz}$ on-frequency masker, and $0.5-\mathrm{kHz}$ off-frequency masker were selected for this experiment, which follow parameters used in earlier studies (e.g., Nelson et al. 2001). The use of higher frequency off-frequency maskers has been proposed to avoid potential compression influences on lower frequency off-frequency maskers (e.g., LopezPoveda et al. 2003). Nonetheless, this selection requires the additional assumption that the rate of recovery from forward masking does not depend on probe frequency. Although the rationale for the selection of masker and probe frequencies for this experiment was sound, the large individual differences seen in the slopes of the offfrequency TMCs call into question the suitability of a $0.5-\mathrm{kHz}$ off-frequency masker for a mid-frequency probe.

Accordingly, the linear-reference TMC (along with several other factors) was investigated in Experiment 2 as a potential source of variance contributing to the large individual differences seen in the results of Experiment 1. In addition to masker and probe frequencies for the linear-reference TMC, other factors included: test-retest reliability of TMCs, effects of probe sensation level, parameters of TMC fitting algorithms, and subject-related variables such as age, strength of cochlear nonlinearities estimated with distortion-product otoacoustic emissions (DPOAEs), and presumed underlying cochlear damage (outer hair cells versus inner hair cells).

\section{EXPERIMENT 2}

Methods

Subjects. Subjects in Experiment 2 included 20 of the 51 subjects who completed Experiment 1; these individuals ranged in age from 21 to 87 years (mean $=57.4$ years; seven males; 13 females). For data analyses, subjects remained in their original threshold groups, as described for Experiment 1: (1) low threshold group $(n=7$; mean age $=37.9$ years, range $=21-67)$; (2) mid threshold group $(n=7$; mean age $=59.3$ years, range $=$ 22-80); and (3) high threshold group $(n=6$; mean age $=$ 77.9 years, range $=68-87)$. Each subsection of Results reports the number of subjects with available data contributing to each analysis.

Stimuli and apparatus. TMCs were measured as in Experiment 1 with a $1.0-\mathrm{kHz}$ probe at $10 \mathrm{~dB}$ SL for onfrequency $(1.0 \mathrm{kHz})$ and off-frequency $(0.5 \mathrm{kHz})$ maskers. Two additional measures were then obtained in random order: (1) a new linear-reference TMC, with a $0.8-\mathrm{kHz}$ off-frequency masker for a $2.0-\mathrm{kHz}$ probe and (2) TMCs with a $1.0-\mathrm{kHz}$ probe at $16 \mathrm{~dB}$ SL for a $1.0-\mathrm{kHz}$ on-frequency masker. Stimuli were delivered using the same apparatus and in the same manner as in Experiment 1. Experiment 2 included measurement of
DPOAEs in addition to TMCs (details in a later section). DPOAE responses were recorded using an Otodynamics Echoport ILO292 USB-II (V6.0) located in an audiometric test booth. The "checkfit" mode ensured that the level and stimulus waveform in the ear canal were appropriate prior to recording a response. Before DPOAEs were measured, normal middle ear status was confirmed by otoscopic examination and tympanometry using a $226-\mathrm{Hz}$ probe tone.

Procedures. TMC measurement and compression estimation procedures were identical to those described in Experiment 1. For the two additional measures obtained in Experiment 2, compression estimates were inferred from (1) the new linear-reference TMC and the $1.0-\mathrm{kHz}$ on-frequency masker and (2) two on-frequency TMCs at two probe sensation levels. Experiment 2 was completed following Experiment 1; therefore, subjects had additional experience from Experiment 1 prior to data collection for this experiment. Time between test sessions in Experiments 1 and 2 varied across subjects, ranging from 1 to 108 weeks (average $=41$ weeks).

Prior to data collection in Experiment 2, thresholds for the $1.0-\mathrm{kHz}$ probe were re-measured to determine if significant changes occurred that would have required changes in probe levels (fixed at $10 \mathrm{~dB}$ above probe threshold). Probe thresholds for trial 1 $($ mean $=31.1 \mathrm{~dB}$ SPL $)$ and trial $2($ mean $=31.3 \mathrm{~dB}$ SPL $)$ were not significantly different $[t(19)=-0.775 ; p=$ $0.448]$; the largest individual difference between trials was $3.3 \mathrm{~dB}$. For consistency, probe thresholds for trial 1 were used to set probe levels and to assess relationships with variables of interest.

DPOAEs (defined as the amplitude of the $2 f_{1}-f_{2}$ response) were measured in half-octave bands for $f_{2}$ primaries between 1.0 and $6.0 \mathrm{kHz}\left(f_{2} / f_{1}=1.22\right) . L_{1}$ and $L_{2}$ were fixed at 65 and $55 \mathrm{~dB}$ SPL, respectively. From these values, DPOAE sum was computed by adding the level of the total DPOAE response in each half-octave band (dB SPL). In addition, DPOAE input-output functions were measured for $f_{2}$ fixed at $1.0,2.0$, and $4.0 \mathrm{kHz}$ and $f_{2} / f_{1}=1.22$. $L_{2}$ ranged from 25 to $75 \mathrm{~dB}$ SPL in 5-dB steps. For $L_{2}$ values of $65-$ $75 \mathrm{~dB}$ SPL, $L_{1}=L_{2}$. For $L_{2}$ values $<65 \mathrm{~dB}$ SPL, $L_{1}$ was determined by the formula, $\mathrm{L}_{1}=0.4 * \mathrm{~L}_{2}+39 \mathrm{~dB}$, which has been shown generally to produce large DPOAE responses in normal-hearing subjects (Kummer et al. 1998). At each $L_{2}$, averaging was stopped manually when the level of the response and the signal-to-noise ratio were stable and a spectral component at the DPOAE frequency was observed above the noise floor. DPOAE amplitude and the level of the noise within the $2 f_{1}-f_{2}$ response bin corresponding to +2 standard deviations above the mean noise level were recorded as a function of $L_{2}$. DPOAEs were recorded from the same ear used to measure TMCs (right ear) and were obtained twice to evaluate test-retest reliability. Time 
between test sessions varied across subjects, ranging from 0 to 70 days (average $=14$ days). If no measurable DPOAEs were obtained in the first test session, no retest was conducted (this occurred in five subjects).

\section{Results and discussion}

Test-retest reliability. Little systematic information is available on the test-retest reliability of compression estimates obtained from TMCs. The amount of practice prior to data collection, stimulus frequencies tested, number of measures for each threshold estimate, and the use of extrapolated data vary among studies (e.g., Plack et al. 2004; Rosengard et al. 2005). Test-retest reliability of forward-masked thresholds is one way of evaluating the precision of inferred basilar membrane input-output responses derived from TMCs for adult subjects of all ages and hearing levels. Here, the aim was to repeat measurements of off-frequency and onfrequency TMCs in a subset of individuals from Experiment 1 to assess test-retest reliability.

Slopes of off-frequency TMCs were available for 19 of 20 subjects in Experiment 2. Figure 4 shows that the slopes of off-frequency TMCs from trials 1 and 2 are strongly positively correlated $(p<0.001)$ and are clustered fairly evenly around the reference line with a slope of 1 . In addition, mean TMC slopes for the two trials $(0.380 \mathrm{~dB} / \mathrm{dB}$ versus $0.396 \mathrm{~dB} / \mathrm{dB})$ did not differ significantly $[t(18)=-1.069 ; p=0.299]$, providing additional evidence of good repeatability of the measured TMC masker levels. Slopes of off-frequency TMCs did not vary significantly with probe threshold for trial 1 $(r=-0.272, p=0.264)$ or trial $2(r=0.049, p=0.845)$, as

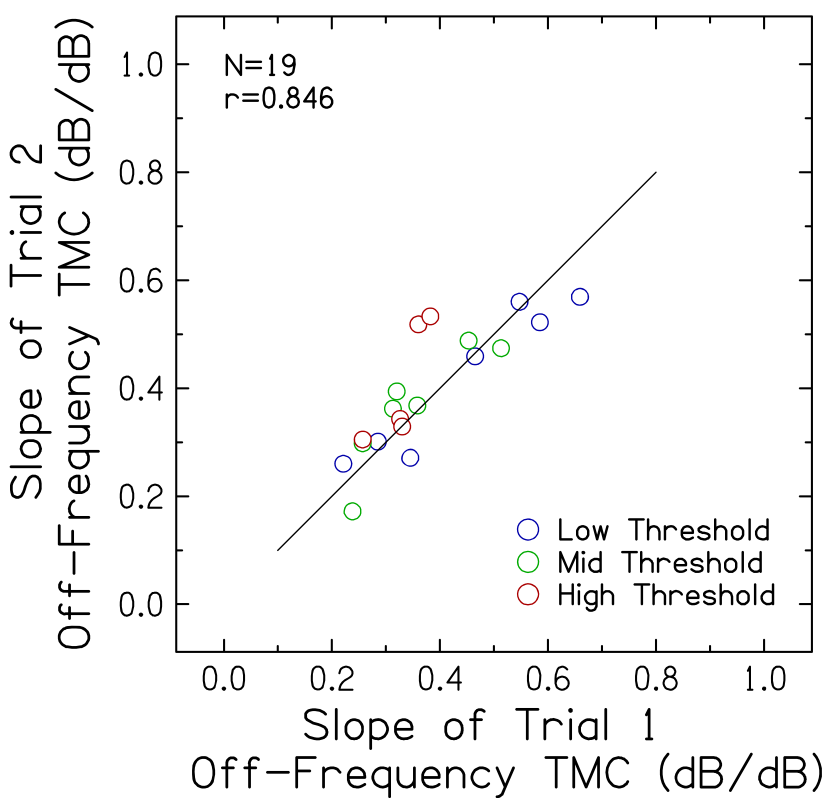

FIG. 4. Slopes of off-frequency TMCs for trial 2 plotted against slopes for trial 1. A line with a slope of 1 is included for reference. was observed for the full set of TMC slopes shown in Figure 3.

Further analysis of test-retest reliability of TMCs was investigated for a subset of subjects $(N=15)$ for whom masker levels were measured for on- and offfrequency maskers at five masker-probe intervals $(0$, 10, 20, 30, and $40 \mathrm{~ms}$ ). Similar to the analysis of TMCs described in Experiment 1, masker levels were assessed using a repeated measures ANCOVA with trial (trials 1 and 2), masker type (on- and offfrequency), and masker-probe interval as within-subjects factors, and probe threshold as a covariate. Because age did not show a significant effect when added as a covariate $[F(1,12)=0.32, p=0.580]$, age was not included in this ANCOVA. Results showed no significant differences between trials 1 and $2[F(1,13)=$ $1.96, p=0.185]$ and no significant interactions with trial. Consistent with results described in Experiment 1, significant interactions were found between masker type and masker-probe interval $[F(4,52)=6.53 ; p<$ 0.001] and among masker type, masker-probe interval, and probe threshold $[F(4,52)=3.13 ; p=0.022]$. The lack of significant interactions of these factors with trial suggests that masker levels and the general shapes of TMCs did not change in a systematic way when remeasured many weeks later.

Linear-reference TMCs using higher frequency signals Effect on slopes of off-frequency TMCs. In Experiment 1, large individual differences were seen in the slopes of off-frequency TMCs, which raised questions about the suitability of the $0.5-\mathrm{kHz}$ off-frequency masker for a mid-frequency probe. The significance of these results relates to the question of the extent to which compression is applied to off-frequency signals, especially for lower frequency probes combined with maskers an octave or less below the probe (e.g,. Lopez-Poveda et al. 2003; Rosengard et al. 2005). As mentioned earlier, Lopez-Poveda and Alves-Pinto (2008) reported that for a $4.0-\mathrm{kHz}$ probe frequency, basilar membrane responses to a $2.2-\mathrm{kHz}$ masker showed approximately 2:1 compression, whereas responses to a $1.6-\mathrm{kHz}$ masker reflected linear basilar membrane responses. The frequencies selected for the probe $(2.0 \mathrm{kHz})$ and off-frequency masker $(0.8 \mathrm{kHz})$ for Experiment 2 were a compromise that included the recommended wider frequency separation (Lopez-Poveda and Alves-Pinto 2008), with probe frequencies low enough for thresholds to be measurable for the current subjects with poorer hearing at higher frequencies.

Thus, in Experiment 2, an additional linear-reference TMC was obtained with a higher frequency offfrequency masker $(0.8 \mathrm{kHz})$ for a higher frequency probe $(2.0 \mathrm{kHz})$. The new linear reference was obtained from 19 of 20 subjects. As in Experiment 1, slopes of the off-frequency TMCs were estimated with straight-line fits 


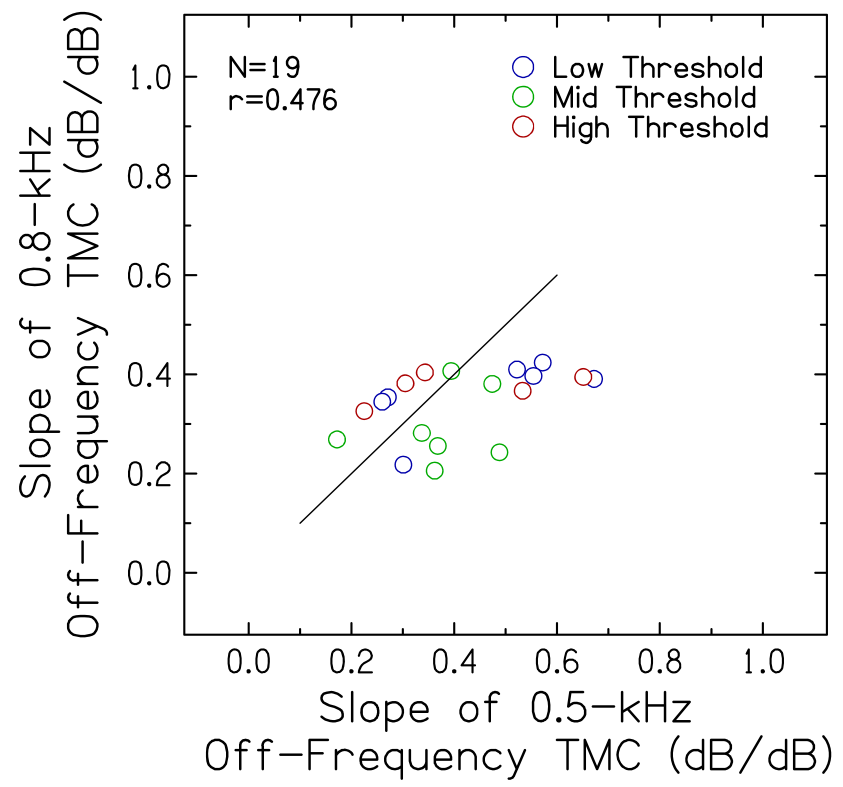

FIG. 5. Slopes of off-frequency TMCs for the $0.8-\mathrm{kHz}$ off-frequency masker plotted against slopes for the $0.5-\mathrm{kHz}$ off-frequency masker. A line with a slope of 1 is included for reference.

for the $0.8-\mathrm{kHz}$ off-frequency masker. Figure 5 shows TMC slopes for the $0.8-\mathrm{kHz}$ off-frequency masker plotted against slopes for the $0.5-\mathrm{kHz}$ off-frequency masker. As indicated by the significant positive correlation $(p=0.038)$, subjects with shallower TMC slopes for the $0.5-\mathrm{kHz}$ masker also had shallower TMC slopes for the 0.8-kHz masker. Mean TMC slopes for the $0.5-\mathrm{kHz}$ masker $(0.41 \mathrm{~dB} / \mathrm{dB})$ were significantly steeper than for the $0.8-\mathrm{kHz}$ masker $(0.34 \mathrm{~dB} / \mathrm{dB})[t(18)=2.41$; $p=0.027]$. Consistent with this finding, the steepest slopes were obtained with the $0.5-\mathrm{kHz}$ masker, as indicated by the placement of data points with respect to the reference line in Figure 5. Some of these slopes were from subjects in the low threshold group (blue), for whom compression was presumed to be normal or near normal. If the slope of the off-frequency TMC reflected both the decay of masking and compression applied to the masker, a steeper slope would have been obtained for this TMC than for a linear reference that reflected only decay of masking. Nevertheless, slopes of $0.5-\mathrm{kHz}$ and $0.8-\mathrm{kHz}$ off-frequency TMCs did not vary significantly with probe threshold ( $r=-0.034$ and 0.149 , respectively; not shown), consistent with results for the $0.5-\mathrm{kHz}$ masker from the larger set of subjects in Experiment 1 (Fig. 3).

Effect on estimates of compression slope. Although slopes of off-frequency TMCs for both masker frequencies $(0.5$ and $0.8 \mathrm{kHz}$ ) did not change significantly with probe threshold, it was of interest to determine the extent to which compression slope estimates varied at the $1.0-\mathrm{kHz}$ place with probe threshold when a higher frequency linear-reference TMC was used. Compression slope estimates were available for 15 of the 20 subjects who participated in Experiment 2. As shown previously in Figure 2 with the larger set of subjects, compression slope computed using the $0.5-\mathrm{kHz}$ off-frequency masker and the $1.0-\mathrm{kHz}$ on-frequency masker increased slightly but significantly with increasing probe threshold. Figure 6 extends those results and displays compression slope computed using 0.5 - and $0.8-\mathrm{kHz}$ off-frequency maskers and the $1.0-\mathrm{kHz}$ on-frequency masker plotted against probe threshold, which shows significant positive correlations for both maskers $(p=0.003$, and $p<0.001$, respectively). Not shown is the strong positive correlation between compression slopes derived from both maskers ( $r=0.837, p<0.001)$; subjects with shallower compression slopes derived with the $0.5-\mathrm{kHz}$ off-frequency masker also have shallower slopes derived with the $0.8-\mathrm{kHz}$ offfrequency masker. Finally, compression slope estimates derived using the two off-frequency maskers and the $1.0-\mathrm{kHz}$ on-frequency masker did not differ significantly $[t(14)=1.05 ; p=0.311]$, suggesting that whatever differences were observed between slopes of the two linear-reference TMCs did not have a systematic effect on compression slope estimates.

In contrast to the results in Figure 2, which showed compression slope increasing only slightly with increasing probe threshold, larger changes in compression slope with increasing threshold are seen in Figure 6. Differences in the magnitude of the effect of hearing loss on compression slope in Experiments 1 and 2 (Fig. 2 versus Fig. 6) may be due to several factors, including larger sample sizes in Experiment 1,

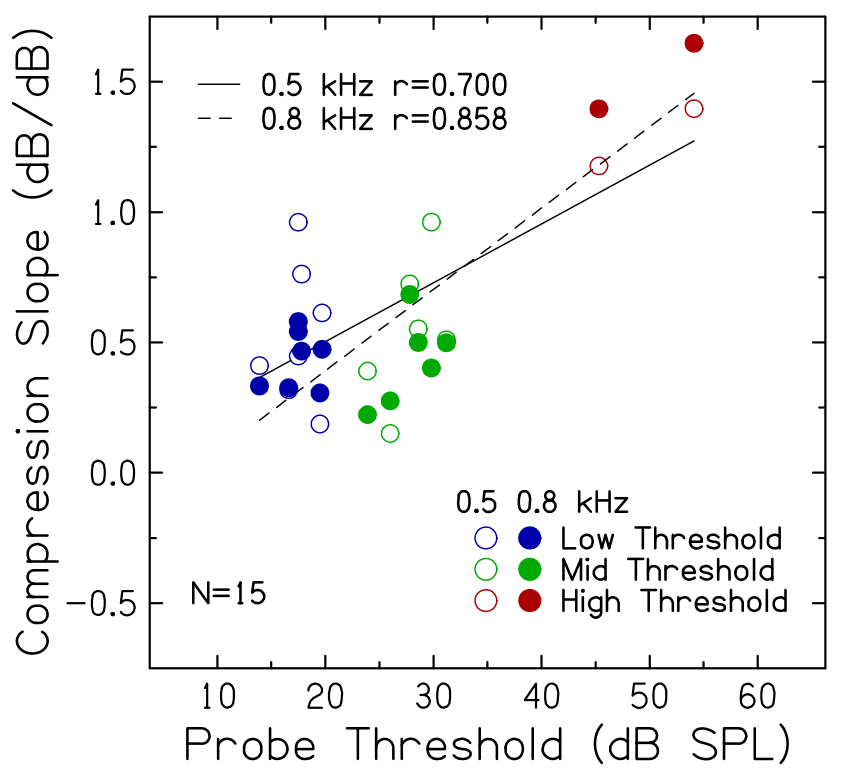

FIG. 6. Compression slope estimates for $0.5-\mathrm{kHz}$ (open) and 0.8$\mathrm{kHz}$ (filled) off-frequency maskers plotted against probe threshold. To minimize variance, inferred input-output responses included all (6 to 8) threshold estimates from trial 1 and trial 2 for each on-frequency masker-probe interval. Pearson correlation coefficients and linear regression functions are included. 
differences in the distribution of probe thresholds (fewer subjects with higher thresholds in Experiment 2), fewer subjects with normal hearing and steep slope estimates, more valid estimates in Experiment 2 as a result of prior experience, or the use of a higher frequency linear reference in Experiment 2. Wherever a comparison of results from Experiments 1 and 2 for the $0.5-\mathrm{kHz}$ off-frequency TMC was available, masker levels did not differ significantly (reviewed in test-retest reliability section and shown in Fig. 4).

In Figure 6, steep compression slopes were estimated for the $0.5-\mathrm{kHz}$ off-frequency masker for the two subjects with the highest probe thresholds (50-60 dB SPL) and even steeper slopes for the $0.8-\mathrm{kHz}$ masker (red symbols). Unusually steep compression slopes for the $0.5-\mathrm{kHz}$ masker are seen for two subjects with probe thresholds $\sim 20 \mathrm{~dB}$ SPL (blue open symbols), with shallower slopes for the same subjects obtained with the $0.8-\mathrm{kHz}$ masker $(0.47-0.58 \mathrm{~dB} / \mathrm{dB})$. These results suggest that basilar membrane compression may have influenced $0.5-\mathrm{kHz}$ off-frequency maskers of $1.0-\mathrm{kHz}$ probes for some subjects. Additional data are needed to determine if more accurate estimates of basilar membrane compression may be obtained using higher frequency maskers and probes.

Effects of probe sensation level. Given that the most appropriate linear reference for the TMC method has not been established, an alternative option is to obtain compression estimates that do not rely on a linearreference TMC. This is of particular interest for assessing compression in subjects with hearing loss, for whom it may not be possible to obtain a linearreference TMC, especially at higher frequencies where hearing loss is greater. In Experiment 2, procedures followed the variant TMC method described by Lopez-Poveda and Alves-Pinto (2008). The variant method allows basilar membrane inputoutput responses to be inferred from two on-frequency TMCs (1.0-kHz probe and $1.0-\mathrm{kHz}$ masker) measured with two low-level, 1.0-kHz probes that differ in sensation level by $6 \mathrm{~dB}$. The method makes two assumptions: (1) the response of the basilar membrane is linear over a range of low probe levels (reviewed by Robles and Ruggero 2001) and (2) the time course of decay of the internal effect of the masker is the same for the two probe levels. Accordingly, with a 6-dB increase in probe level, the corresponding increase in masker level needed for detection threshold should be $6 \mathrm{~dB}$ for linear responses and more than $6 \mathrm{~dB}$ for compressive responses. Thus, compression is estimated by comparing slopes for on-frequency TMCs at the same masker-probe intervals. Lopez-Poveda and Alves-Pinto (2008) reported similar compression estimates for the variant method compared to those obtained with standard methods for subjects with normal hearing.
In Experiment 2, masker levels for two on-frequency TMCs were obtained for probe levels of $10 \mathrm{~dB}$ SL and 16 dB SL. As illustrated in Figure 7 (top) for one representative subject from the low threshold group (LT18), a slope for each on-frequency TMC was calculated from a straight line fit to data (1) that appeared to fall on a relatively straight line and (2) for which thresholds were measured over a similar range of masker levels (within $5 \mathrm{~dB}$ ) for both probe sensation
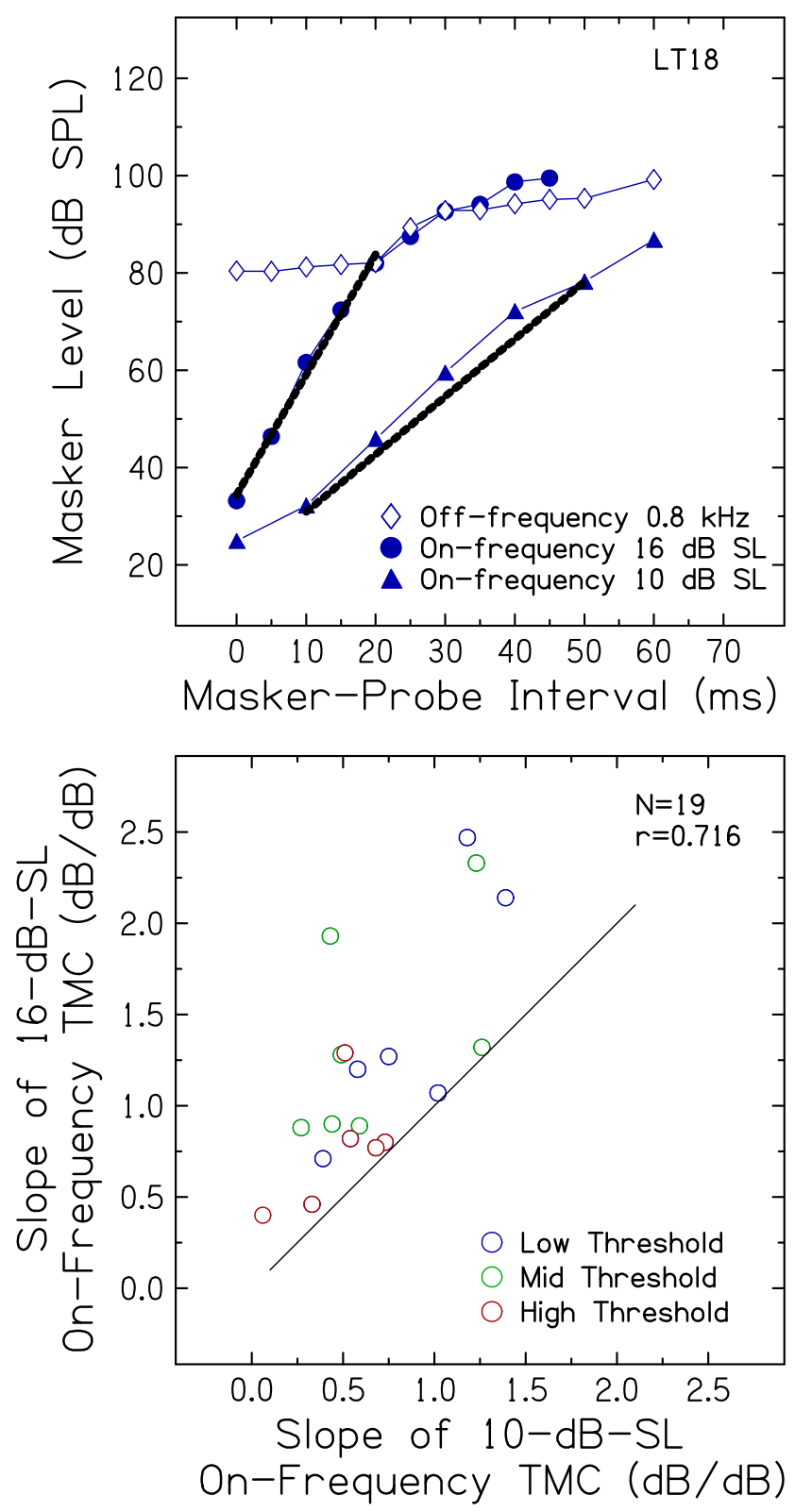

FIG. 7. Top TMCs from one subject in the low threshold group (LT18) showing masker level at threshold as a function of maskerprobe interval for the off-frequency masker $(0.8 \mathrm{kHz}$, diamonds) and on-frequency masker $(1.0 \mathrm{kHz})$ for a $16-\mathrm{dB}-\mathrm{SL}$ probe (circles) and a 10-dB-SL probe (triangles). The thick black lines represent straightline fits for comparison of slopes over a similar range of masker levels. Bottom slopes of on-frequency TMCs for $1.0-\mathrm{kHz}$ probes at $16 \mathrm{~dB}$ SL plotted against slopes for $1.0-\mathrm{kHz}$ probes at $10 \mathrm{~dB}$ SL. A line with a slope of 1 is included for reference. 
levels. Apparent differences in slopes of the two onfrequency maskers at the same masker levels are a concern, given that basilar membrane compression should be the same over the same range of masker levels.

On-frequency TMC measures at two probe sensation levels were available for 19 of the 20 subjects who participated in Experiment 2. In Figure 7 (bottom), slope estimates from TMCs using the 16-dB-SL probe are plotted against slope estimates using the 10-dB-SL probe. Although positively correlated $(p=0.001)$, TMC slopes for the higher sensation level probe were significantly steeper $($ mean $=1.21 \mathrm{~dB} / \mathrm{dB})$ than those for the lower sensation level probe $($ mean $=0.68 \mathrm{~dB} / \mathrm{dB})$ $[t(17)=-5.41 ; p<0.001]$. Indeed, slopes of on-frequency TMCs for all 19 subjects were steeper for the higher level probe than for the lower level probe, as indicated by the placement of data points with respect to the reference line in Figure 7. No significant association was observed between this TMC slope difference and age or probe threshold. Lopez-Poveda and Alves-Pinto (2008) discussed a similar finding in their data and suggested that the difference in slopes of on-frequency TMCs was due to the change in the rate of decay of masking with increasing masker-probe interval. However, similar changes in slope were not apparent in the masker levels in the off-frequency TMCs from their 2008 study or from results of the current study (see top panel of Fig. 7, for example). An alternative explanation is that the lower sensation level probe may not be as salient as the higher sensation level probe, resulting in increased confusion and less steep slopes for some subjects, which thereby underestimates compression. These results suggest that the use of a lower sensation level probe (such as $10 \mathrm{~dB}$ SL) may contribute to individual differences in compression estimates using the TMC method. More research is needed to provide a systematic examination of these effects, for example, TMCs measured for probes at several sensation levels for an on-frequency masker.

Parameters of TMC fitting algorithms and underlying assumptions. The selection of parameters for TMC fitting algorithms was also considered a potential source of variance contributing to individual differences in Experiment 1. For example, one criterion for including an estimate of inferred input-output response was rms error between fitted and measured values $<5 \mathrm{~dB}$. Figure 8 (top) shows compression slope estimates plotted against rms error for the 43 subjects from Experiment 1 (43 of 51 subjects with inferred input-output responses). Compression slope systematically increased as rms error increased $(r=0.511, p<0.001)$. Some of the largest rms error values were for subjects in the low threshold group (blue), who presumably have normal or near normal compression. No significant correlations were observed between rms error and probe threshold or subject age ( $r=-0.091$ and $r=0.174$, respectively), which rules out poorer hearing or older listeners as sources of the less- than-optimal fits. This result suggests that the accuracy of the fits of inferred basilar membrane input-output responses may contribute to individual differences in estimates of compression slope derived using the TMC method.

A stricter criterion of $\mathrm{rms}$ error $<3 \mathrm{~dB}$ for including an estimate of compression slope was also considered. For the 31 estimates meeting this criterion, compression slope did not change significantly with rms error $(r=0.282)$, but still increased significantly with increasing probe threshold $(r=0.397, p=0.026)$, as shown in Figure 8 (bottom). Filled symbols in the bottom panel are compression slope estimates with rms errors ranging from 3 to $5 \mathrm{~dB}$, which illustrate that less-than-optimal fits occurred more often when compression slope estimates were relatively high, but occurred for subjects with a range of probe thresholds. Indeed, the slope of the regression indicating the magnitude of the effect of hearing loss on compression slope does not appear to be influenced by rms error $(0.0088$ and $0.0084 \mathrm{~dB} / \mathrm{dB}$ for the entire group and for the group with $<3 \mathrm{~dB}$ rms error, respectively).

Another issue related to assumptions underlying the use of TMCs to infer basilar membrane inputoutput responses is the effect of the MOCR. With an onset delay estimated at $\sim 25 \mathrm{~ms}$ (Backus and Guinan 2006; Guinan 2006), the MOCR may decrease the gain of the active cochlear mechanism under certain conditions (e.g., Jennings et al. 2009). Depending on the duration of the masker, the activation of the MOCR may complicate interpretation of TMCs if forward-masked thresholds measured at shorter masker-probe intervals reflect a different cochlear process than thresholds measured at longer intervals. Relevant to the current experiment, the MOCR may contribute to individual differences to the extent that characteristics of the MOCR vary among individuals with similar hearing sensitivity (Backus and Guinan 2007; Lilaonitkul and Guinan 2009), as in the notion of "tough" and "tender" ears related to noise susceptibility (Maison and Liberman 2000). The MOCR was not assessed in the current experiment; additional research is necessary to determine the extent to which the MOCR affects compression estimates inferred from TMCs and contributes to individual differences in these measures (Wojtczak and Oxenham 2010).

Subject age. Another source of individual differences in behavioral estimates of compression is subject age. Age-related declines in temporal processing have been demonstrated for several measures (e.g., Strouse et al. 1998). However, relevant to the current experiment, Gifford and Bacon (2005) found no consistent agerelated differences in measures related to cochlear nonlinearities (auditory filter widths, suppression, and growth of forward masking). Higher forward-masked thresholds or lower masker levels at threshold have been 

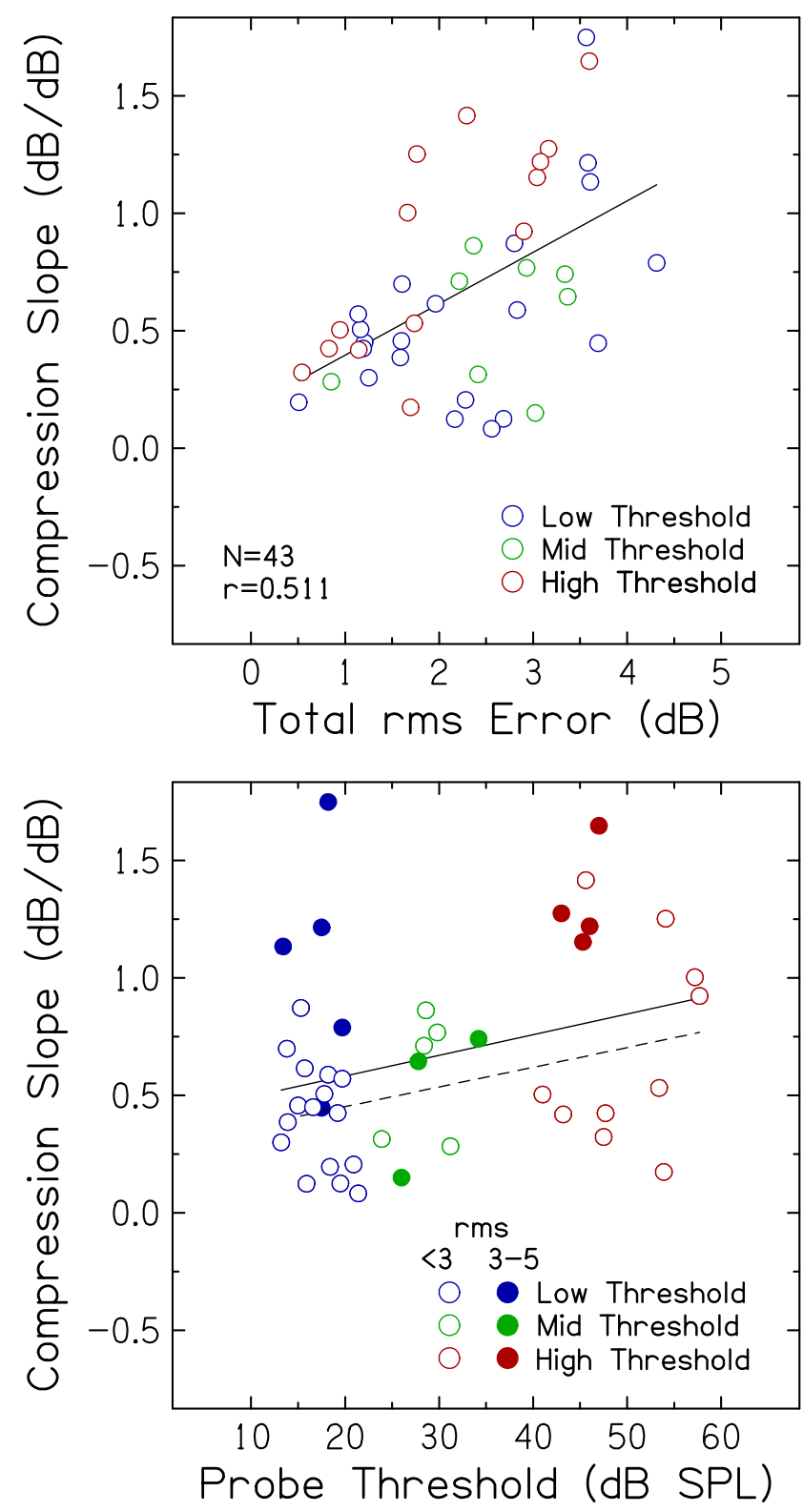

FIG. 8. Top compression slope estimates plotted against rms error, for input-output functions fitted with rms errors $<5 \mathrm{~dB}$. The Pearson correlation coefficient and linear regression function are included. Bottom compression slope estimates plotted against probe threshold. Open symbols represent fits with rms errors $<3 \mathrm{~dB}$. Filled symbols represent fits with rms errors ranging from 3 to $5 \mathrm{~dB}$. The solid line is the linear regression function including all data (same as Figure 2, top); the dashed line is the linear regression function including compression slope estimates with rms errors $<3 \mathrm{~dB}$.

observed for older than younger subjects (Sommers and Gehr 1998; Gifford and Bacon 2005; Gifford et al. 2007; Dubno et al. 2002). In some cases, these age-related differences were observed across a wide range of masker-probe intervals and may relate to higher detection thresholds for older than younger subjects (Dubno et al. 2003). Age-related differences in amount of forward masking (rather than in rate-of-decay of forward masking) may or may not represent a confounding factor for the TMC method, given the importance to the method of how masker levels change with increasing masker-probe interval for on- and offfrequency maskers.

Although measurement of temporal processing was not a goal of this experiment, it was of interest to determine the extent to which this factor accounts for any age-related differences in compression estimates derived using the TMC method (which is to some extent a temporal measure). For measurement of TMCs, detection thresholds for the 20-ms probe and the 200-ms on-frequency masker at $1.0 \mathrm{kHz}$ were available for 47 of 51 subjects. Threshold differences for these two signals provided an estimate of temporal integration, a measure of temporal processing (see review by Reed et al. 2009). For subjects with normal hearing, thresholds typically decrease with increasing signal duration from 10 to $200 \mathrm{~ms}$; a doubling of signal duration corresponds to $\sim 3$-dB decrease in threshold. Larger decreases in thresholds with increasing signal duration correspond to steeper temporal integration slopes. For the 47 subjects with available data, temporal integration (threshold difference between 20-ms and 200-ms signals) ranged from 1.7 to $17.4 \mathrm{~dB}$.

Temporal integration was negatively correlated with age $(r=-0.395, p<0.001)$ and with threshold for the 200-ms signal $(r=-0.664, p<0.001)$; younger subjects and those with better 200-ms thresholds had steeper temporal integration slopes. Path analysis using Mplus separately assessed threshold and age effects on temporal integration. Threshold for the 200-ms signal accounted for $59 \%$ of the variance in temporal integration $(p<0.001)$, consistent with previous findings showing shallower slopes of temporal integration for subjects with cochlear hearing loss than for subjects with normal hearing (e.g., Florentine et al. 1988). In contrast, age accounted for only $2 \%$ of the variance in temporal integration $(p=0.339)$. These results provide indirect evidence that age-related differences in temporal processing (at least as assessed by temporal integration) may not represent a confounding factor for compression estimates derived using the TMC method. It also suggests that any influence of compression on temporal integration (as suggested by Oxenham et al. 1997 and further evaluated by Plack and Skeels 2007 for shorter duration signals) would not have been confounded by age effects.

To assess the effect of age on TMC estimates of compression, compression slopes and breakpoints for 43 subjects who participated in Experiment 1 were submitted to an ANCOVA, with threshold as a grouping factor and age as a covariate. As reviewed in Experiment 1 , age did not account for a significant portion of the variance in compression slope or breakpoint. A path analysis using Mplus revealed that, as expected, age contributed $47.3 \%$ of the variance in probe thresholds 
$(p<0.001)$, but age uniquely accounted for only $0.2 \%$ of the variance in compression slope $(p=0.825)$ and $0.5 \%$ of the variance in breakpoint $(p=0.450)$. Similarly, age accounted for only $7.7 \%$ of the variance in the slopes of off-frequency TMCs $(p=0.870)$. Finally, as reported earlier in two ANCOVAs with age as a covariate, masker levels at threshold for off-frequency and on-frequency forward maskers did not differ significantly as a function of age. Taken together, these results provide strong evidence that age per se (independent of age effects on detection thresholds) did not contribute to TMCs or compression measures derived from TMCs.

Cochlear nonlinearities estimated with distortion-product otoacoustic emissions. The TMC method provides estimates of compression characteristics for human subjects measured psychophysically. Although physiological measures of cochlear compression are not available from human subjects, objective measures such as DPOAEs can provide some information to assess qualitatively the accuracy of compression slope estimates derived from TMCs. That is, an evaluation of accuracy using current techniques is based on the degree to which TMC-based estimates of compression are correlated with compression estimates derived from physiologic or objective measures in humans known to reflect cochlear compression, such as DPOAEs. Strong correlations between these measures provide some evidence that individual differences in behavioral estimates of compression reflect true differences among subjects, rather than confounding effects of poor reliability, inappropriate linear-reference TMCs, probe sensation levels, questionable assumptions underlying fitting algorithms, MOCR effects, or subject age, as reviewed earlier.

TMCs and DPOAE input-output functions provide generally similar estimates of basilar membrane responses, given that both are characterized by a nearly linear segment for low input levels and a compressive segment for moderate input levels (e.g., Dorn et al. 2001; Neely et al. 2003; Williams and Bacon 2005). The correlation between basilar membrane input-output responses inferred from TMCs and DPOAEs has been explored in several studies, including Johannesen and Lopez-Poveda (2008) for subjects with normal hearing and Lopez-Poveda and Johannesen (2010) for subjects with hearing loss. Reasonably high within-subject correlations between TMC and DPOAE compression estimates are observed for subjects with normal hearing, especially at high frequencies. Correlations are much less robust for subjects with hearing loss.

To assess the relationship between compression slope estimates derived from TMCs and DPOAEs, DPOAE levels were recorded from 13 subjects in Experiment 2 from the low threshold and mid threshold groups; DPOAEs could not be recorded from subjects in the high threshold group. DPOAE levels and input-output functions were recorded on two occasions to assess test-retest reliability. DPOAE input-output slopes at $1.0 \mathrm{kHz}(N=6), 2.0 \mathrm{kHz}(N=$ $12)$, and $4.0 \mathrm{kHz}(N=9)$ were estimated with straightline fits to DPOAE levels for $L_{2}$ between 40 and $65 \mathrm{~dB}$ SPL, similar to the procedure used by Kummer et al. (1998) and Williams and Bacon (2005).

With regard to test-retest reliability, no significant differences in DPOAE sums were found between trial 1 $($ mean $=14.7 \mathrm{~dB}$ SPL $)$ and trial $2($ mean $=14.0 \mathrm{~dB}$ SPL $)$ $[t(11)=0.845 ; p=0.416]$. No significant differences between input-output slopes for trials 1 and 2 were found using paired two sample for means $t$ tests at $1.0 \mathrm{kHz}(p=0.117), 2.0 \mathrm{kHz}(p=0.745)$, and $4.0 \mathrm{kHz}(p=$ $0.991)$. Based on these findings, DPOAE sums and input-output slopes for trial 1 were used to assess relationships between DPOAE values, probe thresholds, and compression slopes derived from TMCs.

DPOAE sum plotted against probe threshold is shown in Figure 9 (top). As expected, a significant negative correlation between DPOAE sum and probe threshold was observed such that subjects with better hearing, and presumably more robust cochlear nonlinearities, had larger DPOAEs $(p=0.017)$. Figure 9 (bottom) shows slopes of DPOAE input-output functions at $2.0 \mathrm{kHz}$ plotted against compression slopes estimated from the inferred input-output responses using the alternative linear-reference TMC $(0.8 \mathrm{kHz})$; not enough data were available to conduct meaningful correlational analyses for DPOAE functions at $1.0 \mathrm{kHz}$ and TMCs. A non-significant trend $(r=0.596$; $p=0.092$ ) was observed for DPOAE input-output slopes at $2.0 \mathrm{kHz}$ to increase with increasing TMC compression slopes, as expected if both measures reflect the same underlying cochlear nonlinearities, although the data point with the highest slope may be driving this result.

The relatively weak positive correlations between DPOAEs and TMC slopes may relate to the small sample sizes for DPOAEs or may reflect weak evidence of the accuracy of compression estimates from TMCs and/or DPOAEs. DPOAE slopes are generally lower than slopes estimated from TMCs and closer to physiological estimates from animals. At best, analysis of the relationship between DPOAE and basilar membrane inputoutput functions should be considered a qualitative assessment of accuracy because of possible influences of the interference between multiple generating sources of the DPOAE (and resulting fine structure in DPOAE responses), which is not optimal for assessment of basilar membrane compression (Shera and Guinan 1999; Shaffer et al 2003). In addition, although levels for $L_{1}$ and $L_{2}$ are typically set by the Kummer formula because it produces large DPOAE responses for normalhearing subjects, this approach may not be optimal for reflecting compression, especially for hearing-impaired 

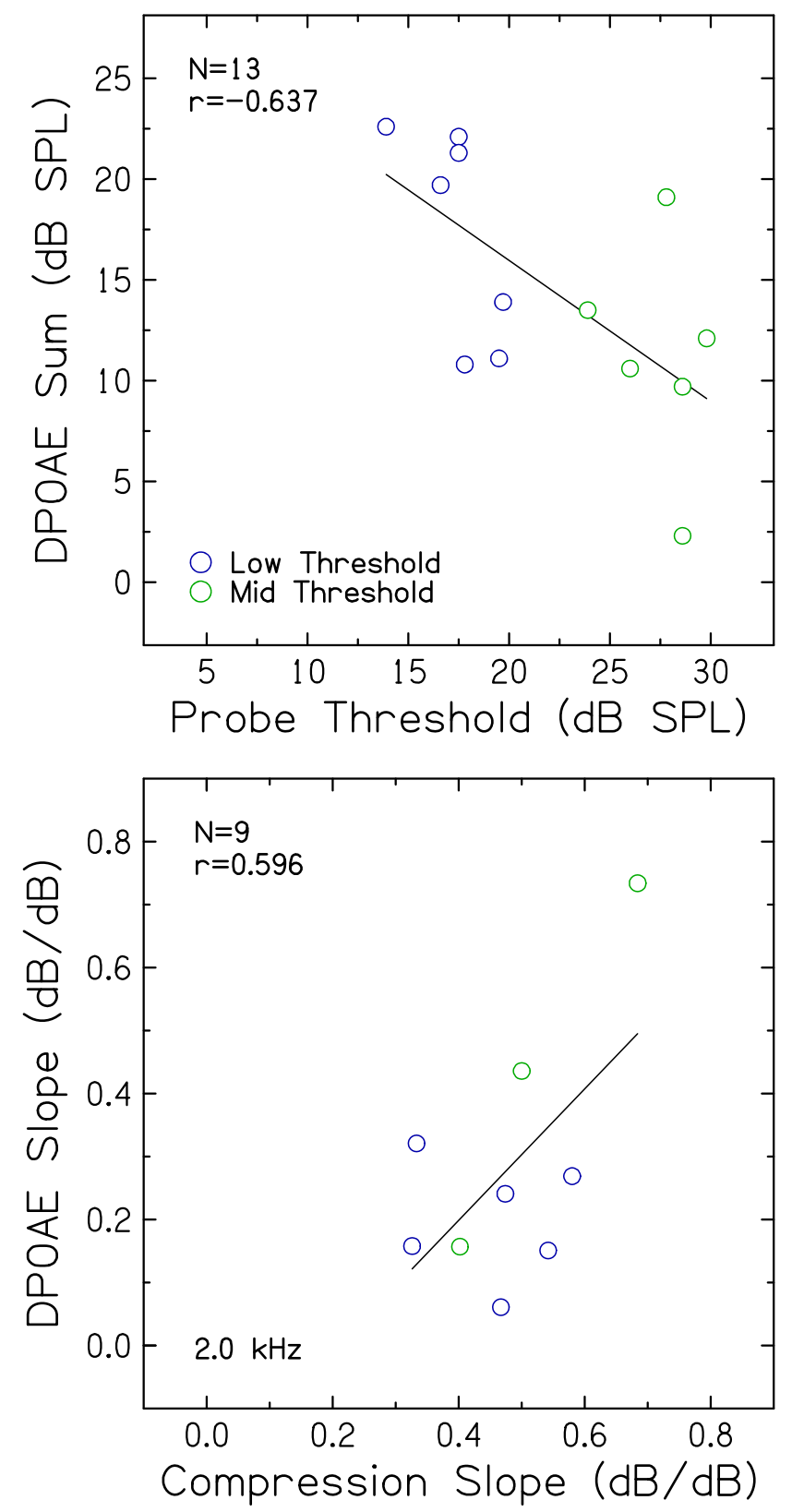

FIG. 9. Top DPOAE sum levels plotted against probe thresholds. Bottom slopes of DPOAE input-output functions at $2.0 \mathrm{kHz}$ plotted against compression slope estimates. Pearson correlation coefficients and linear regression functions are included in each panel.

subjects with reduced compression. These and other factors unrelated to basilar membrane compression may affect the shape of DPOAE input-output functions and impact the strength of the correlations.

Underlying cochlear damage (Outer hair cells versus Inner hair cells). Large differences in compression estimates among individuals with similar degrees of hearing loss may relate to relative differences in damage to outer hair cells (OHCs) and inner hair cells (IHCs) underlying their hearing loss (Moore et al. 1999; Plack et al. 2004; Lopez-Poveda et al. 2005; Lopez-Poveda et al. 2009; Jepsen and Dau 2011;
Howgate and Plack 2011). That is, threshold elevation (in $\mathrm{dB}$ ) for one individual may derive primarily from OHC damage, whereas a similar threshold elevation for another individual may result from damage to both OHCs and IHCs. Briefly, animal studies that selectively damage OHCs or IHCs have shown that OHC damage results in elevated thresholds, reduction in cochlear compression, broadened tuning, and loss of two-tone suppression, which all relate to the cochlear amplifier; in contrast, selective damage to IHCs results in elevated thresholds but normal tuning (for a recent review, see Heinz 2010). Moreover, the distribution of OHC and IHC damage that contributes to the total hearing loss can vary considerably among individuals, due to differences in etiology or noise exposure history (e.g., Liberman and Dodds 1984).

These factors represent potential sources of individual differences in compression slope estimates, to the extent that individuals with similar thresholds and relatively more IHC damage may have more normal compression. This is based on the assumption that IHC damage affects the mechano-electrical transduction process and not the cochlear amplifier or the properties of the basilar membrane response, such that the shape of the basilar membrane inputoutput function is similar for individuals whose hearing loss results from pure IHC damage and individuals with normal hearing. However, the basilar membrane response necessary to reach detection threshold must be larger to offset the change in the transduction process. Thus, the basilar membrane response to the probe would be larger at detection threshold for individuals with IHC damage compared to those with OHC damage. Because the basilar membrane response to the off-frequency masker is assumed to be linear at the probe place, a higher masker level would be required at behavioral threshold with IHC damage compared to OHC damage.

Lopez-Poveda et al. (2005) discussed two subjects with very similar detection thresholds but substantially different compression estimates and suggested that relative differences in IHC and OHC damage for these two subjects could explain these results. Referring to evidence from the auditory nerve that sensitivity at the $\mathrm{CF}$ and in the low-frequency tuning-curve tail regions (e.g., Liberman et al. 1986) is reduced by IHC damage resulting in elevated tuning curves, Lopez-Poveda et al. (2005) suggested a method to estimate this effect comparing the difference in off-frequency TMCs at a short masker-probe interval for subjects with normal and impaired hearing. Howgate and Plack (2011) used a similar approach to estimate the portion of a temporary threshold shift due to OHC and IHC damage. These authors hypothesized that cases with IHC damage would result in off-frequency TMCs with higher masker levels as compared to cases without IHC damage; the difference 
in masker levels provides an estimate of the loss (in dB) due to IHC damage. Finally, Lopez-Poveda and Johannesen (2010) explored this question further with the TMC method by estimating the portion of the total hearing loss (in $\mathrm{dB}$ ) that could be attributed to IHC loss (in $\mathrm{dB}$ ); estimates were taken from subjects' offfrequency masker levels measured for a 2-ms maskerprobe interval, for which it is assumed that higher masker levels correspond to more IHC damage.

To conduct a similar analysis, off-frequency masker levels for the 0-ms masker-probe interval from the large dataset of Experiment 1 were plotted against probe threshold (Fig. 10, top). A \pm 2 standard devia-

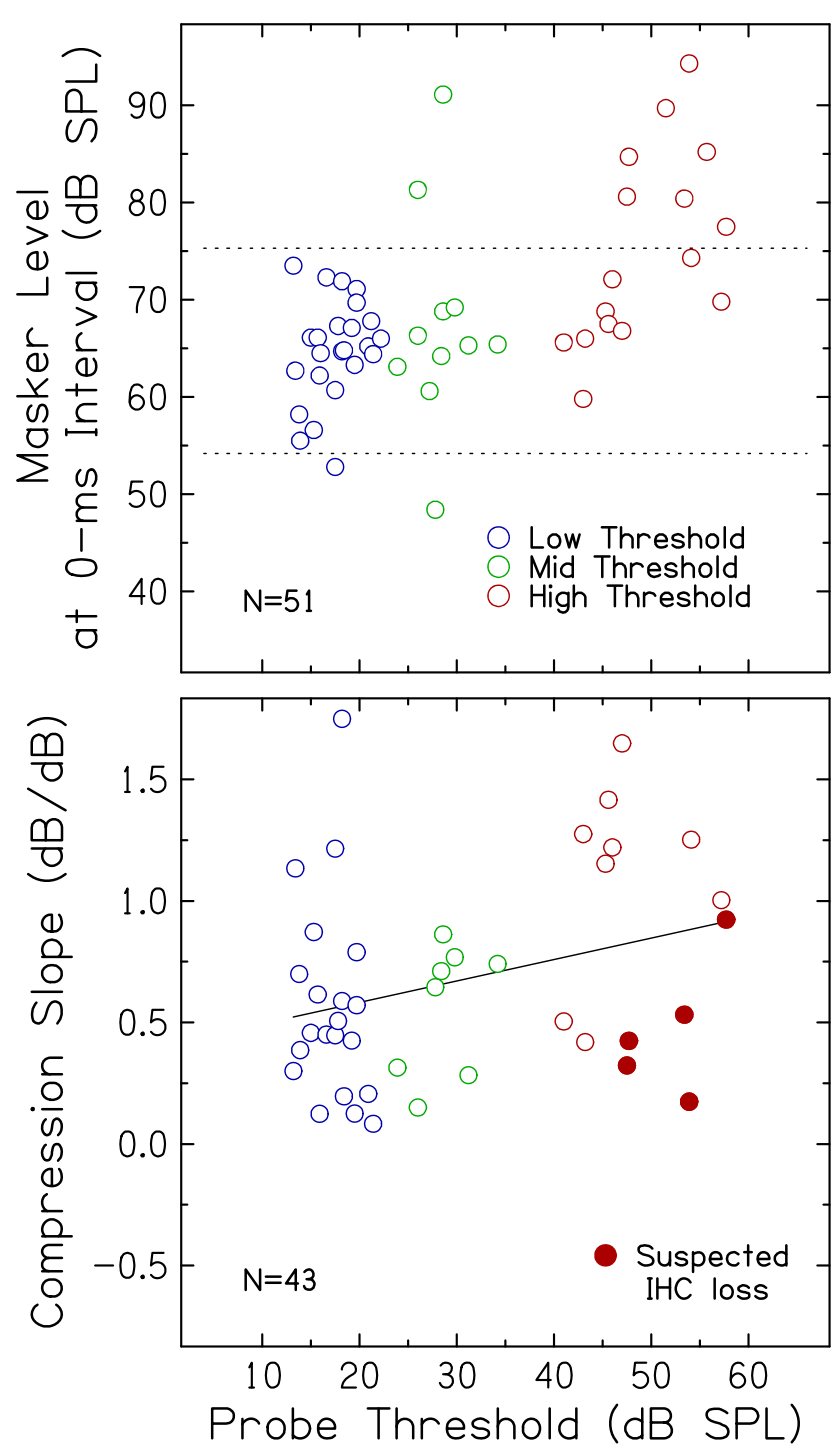

FIG. 10. Top off-frequency masker levels at the 0-ms masker-probe interval plotted against probe threshold. Horizontal dashed lines define the \pm 2 standard deviation range around the masker levels for the low threshold (normal hearing) group. Values above this range suggest IHC damage. Bottom compression slope estimates plotted against probe threshold (same as Fig. 2, top), with filled circles representing suspected cases of IHC damage that also had compression slope estimates. tion range of masker levels for the low threshold (normal hearing) group is indicated by the horizontal dashed lines; masker levels above this range (nine of 51 subjects) were interpreted as reflecting relatively more IHC loss. Because DPOAEs were not measurable in subjects in the high threshold group, it was assumed that these subjects with significantly elevated thresholds had at least some OHC loss. Figure 10 (bottom) replots compression slope estimates for the 43 subjects from Experiment 1 included in Figure 2. Filled circles represent the five presumed cases of IHC damage that also had compression slope estimates. The results are consistent with the assumption that individuals with relatively more IHC damage have more residual compression than others with similar degrees of hearing loss. These results also suggest that the underlying damage contributing to threshold elevation (OHC versus IHC) may represent another source of variance that contributes to large individual differences in compression estimates derived from behavioral measures such as TMCs.

In addition to differential effects of OHC and IHC damage for a particular total hearing loss, differences in underlying cochlear pathology contributing to threshold elevation for various etiologies of sensorineural hearing loss (e.g., strial versus sensory pathology) may also contribute to individual differences in compression, to the extent that nonlinearities are better preserved with certain cochlear pathologies than others (Schmiedt et al. 1990; Schmiedt et al. 2002; Lang et al. 2010). Moreover, detection thresholds and DPOAEs may remain normal despite degeneration of spiral ganglion neurons following noise exposures that result in temporary threshold shifts (Kujawa and Liberman 2009). An additional complicating factor is that auditory-nerve fiber rate-level functions can differ for cases wherein hearing loss results from OHC damage, IHC damage, or combined damage (Heinz and Young 2004). Noiseinduced hearing loss has been shown to produce auditory-nerve fibers with slower recovery from adaptation (Scheidt et al. 2010). These results could affect interpretation of TMCs, which assume that differences in decay of forward masking for individuals with normal and impaired hearing relate only to differences in compression. At the very least, it is clear from physiological evidence that detection thresholds for pure tones measured behaviorally do not provide the best estimates of the condition of the inner ear and the strength of nonlinearities for human subjects.

\section{GENERAL DISCUSSION}

The primary goal of the current study was to characterize cochlear nonlinearities using the TMC method in a large sample of adults of various ages whose hearing ranged from normal to moderate cochlear hearing loss. 
Measurement and fitting procedures were selected based on available data from the literature. Compression slope estimates increased slightly but significantly with increasing probe thresholds, consistent with a reduction in compression with increasing hearing loss. An unexpected finding was the large individual differences in estimates of compression slopes, especially for individuals with similar thresholds (Experiment 1). This factor complicated the interpretation of nonlinearities for individuals with normal and impaired hearing. As a result, attention turned to exploring potential sources of variance contributing to individual differences (Experiment 2). Given the conflicting findings in previous studies and the large individual differences obtained in the current study, the effect of hearing loss on estimates of cochlear nonlinearities remains unclear. Although slopes of off-frequency TMCs are assumed to depend only on decay of masking, they have also been shown in some studies to vary significantly with hearing loss or frequency. The possibility of compression influences on the linear-reference TMC and effects of probe sensation level were also considered. Test-retest reliability of TMCs suggested that TMC masker levels and the general shapes of TMCs did not change in a systematic way when re-measured many weeks later. The current results also suggest that age is not a significant contributor to estimates of cochlear nonlinearities, whereas the relative contribution of $\mathrm{OHC}$ and IHC damage may play an important role.

With regard to across-subject variability, it is not known the extent to which differences in compression estimates within and across studies can be explained by measurement factors (which would have negative consequences for behavioral estimates of compression) or reflect true differences in compression among individuals (which could lead to improved characterization of auditory function for individuals and a better understanding of underlying mechanisms). Variability related to measurement noise could relate to (1) the choice of the frequency and frequency separation of the probe and the offfrequency masker and suitability of the linear-reference TMC, (2) the choice of fitting algorithms and assumptions underlying the behavioral estimates, (3) MOCR effects that vary as a function of level and/or maskerprobe interval, (4) changes due to learning or practice that affect data obtained during the course of an experiment, (5) test-retest variability, and (6) influences of confusion or salience that could differentially affect detection of the probe at various masker-probe intervals and TMC slopes. Across-subject variability reflecting true differences among individuals could relate to (1) magnitude of sensorineural hearing loss and the role of OHC and IHC damage, (2) etiology of sensorineural hearing loss (e.g., strial versus sensory loss underlying cochlear pathology), (3) differences in related cochlear nonlinearities (e.g., level-dependent changes in tuning, masking, and DPOAEs), and (4) differences related to changes in auditory-nerve responses with hearing loss. Some of these sources of variability are appropriate in both categories, in that they may provide information about underlying mechanisms but also complicate interpretation of behavioral or objective measures of compression or limit the assumptions underlying these approaches (e.g., MOCR effects and changes in the time course of recovery from adaptation of auditory-nerve fibers).

Unfortunately, most or all of these factors lack reliable assessment methods. This is further complicated by inconclusive investigations that aim to determine the most appropriate measurement and compression estimation procedures. Each factor requires additional research to clarify effects of elevated hearing thresholds and cochlear damage, preferably with large sample sizes that include subjects with varying degrees of hearing loss. These studies should continue the development and testing of methods to account for MOCR effects, estimate and differentiate OHC and IHC damage and other differences in cochlear pathology in human subjects, and determine their relative contributions to individual differences in estimates of basilar membrane compression. Correlations between behavioral measures and various physiological mechanisms are needed for subjects with normal hearing and then extended to subjects with sensorineural hearing loss associated with various etiologies. This information is necessary for the development of improved behavioral or objective measures of cochlear nonlinearities for laboratory and diagnostic/rehabilitative environments. Although it is very likely that information about cochlear function beyond the pure-tone audiogram will provide added benefit for diagnosis and rehabilitation, such as for hearing-aid design and programming, the links between behavioral or objective measures of cochlear function and improved hearing-aid outcomes remain to be established.

\section{ACKNOWLEDGMENTS}

This work was supported (in part) by grants R01 DC00184 and P50 DC00422 from NIH/NIDCD, with a supplement from the American Recovery and Reinvestment Act. The project also received support from the South Carolina Clinical and Translational Research (SCTR) Institute, with an academic home at the Medical University of South Carolina, NIH/NCRR Grant number UL1 RR029882. This investigation was conducted in a facility constructed with support from Research Facilities Improvement Program Grant number C06 RR14516 from the National Center for 
Research Resources, National Institutes of Health. The authors thank Fu-Shing Lee, Keeley McKelvey, and Bryant Mersereau for assistance with data collection and analysis and two anonymous reviewers for helpful comments on the original manuscript. The authors appreciate the sharing of Matlab files used for analysis by Christopher Plack.

\section{REFERENCES}

Backus BC, Guinan JJ (2006) Time-course of the human medial olivocochlear reflex. J Acoust Soc Am 119:2889-2904

Backus BC, Guinan JJ (2007) Measurement of the distribution of medial olivocochlear acoustic reflex strengths across normalhearing individuals via otoacoustic emissions. J Assoc Res Otolaryngol 8:484-496

Dorn PA, Konrad-Martin D, Neely ST, Keefe DH, Cyr E, Gorga MP (2001) Distortion product otoacoustic emission input/output functions in normal-hearing and hearing-impaired human ears. J Acoust Soc Am 110:3119-3131

Dubno JR, Horwitz AR, Ahlstrom JB (2002) Benefit of modulated maskers for speech recognition by younger and older adults with normal hearing. J Acoust Soc Am 111:2897-2907

Dubno JR, Horwitz AR, Ahlstrom JB (2003) Recovery from prior stimulation: masking of speech by interrupted noise for younger and older adults with normal hearing. J Acoust Soc Am 113:2084-2094

Dubno JR, Horwitz AR, Ahlstrom JB (2007) Estimates of basilarmembrane nonlinearity effects on masking of tones and speech. Ear Hear 28:2-17

Florentine M, Fastl H, Buus S (1988) Temporal integration in normal hearing, cochlear impairment, and impairment simulated by masking. J Acoust Soc Am 84:195-203

GIFFORD RH, BACON SP (2005) Psychophysical estimates of nonlinear cochlear processing in younger and older listeners. J Acoust Soc Am 118:3823-3833

GIFFORD RH, BACON SP, Williams EJ (2007) An examination of speech recognition in a modulated background and of forward masking in younger and older listeners. J Speech Lang Hear Res 50:857-864

Guinan JJ (2006) Olivocochlear efferents: anatomy, physiology, function, and the measurement of efferent effects in humans. Ear Hear 27:589-607

HeInz MG (2010) Computational modeling of sensorineural hearing loss. In: Meddis R, Lopez-Poveda E, Popper A, Fay R (eds) Computational models of the auditory system. Springer, New York, pp 177-202

HeInZ MG, Young ED (2004) Response growth with sound level in auditory-nerve fibers after noise-induced hearing loss. J Neurophysiol 91:784-795

Horwitz AR, Ahlstrom JB, Dubno JR (2007) Speech recognition in noise: estimating effects of compressive nonlinearities in the basilar-membrane response. Ear Hear 28:682-693

Horwitz AR, AhLstrom JB, Dubno JR (2011) Level-dependent changes in detection of temporal gaps in noise markers by adults with normal and impaired hearing. J Acoust Soc Am, in press

Howgate S, Plack CJ (2011) A behavioral measure of the cochlear changes underlying temporary threshold shifts. Hear Res 277:78-87

Jennings SG, Strickland EA, Heinz MG (2009) Precursor effects on behavioral estimates of frequency selectivity and gain in forward masking. J Acoust Soc Am 125:2172-2181

JePSEN ML, DAU T (2011) Characterizing auditory processing and perception in individual listeners with sensorineural hearing loss. J Acoust Soc Am 129:262-281
Johannesen PT, Lopez-Poveda EA (2008) Cochlear nonlinearity in normal-hearing subjects as inferred psychophysically and from distortion-product otoacoustic emissions. J Acoust Soc Am 124:2149-2163

KujaWa S, Liberman MC (2009) Adding insult to injury: cochlear nerve degeneration after "temporary" noise-induced hearing loss. J Neurosci 29:14077-14085

Kummer P, Janssen T, ARnold W (1998) The level and growth behavior of the $2 f_{1}-f_{2}$ distortion product otoacoustic emission and its relationship to auditory sensitivity in normal hearing and cochlear hearing loss. J Acoust Soc Am 103:3431-3444

Lang H, Jyothi V, Smythe NM, Dubno JR, Schulte BA, Schmiedt RA (2010) Chronic reduction of endocochlear potential reduces auditory nerve activity: further confirmation of an animal model of metabolic presbyacusis. J Assoc Res Otolaryngol 11:419-434

LevitT H (1971) Transformed up-down methods in psychoacoustics. J Acoust Soc Am 49:467-477

Liberman MC, DodDs LW (1984) Single-neuron labeling and chronic cochlear pathology. III. Stereocilia damage and alterations of threshold tuning curves. Hear Res 16:55-74

Liberman MC, Dodds LW, Learson D (1986) Structure-function correlation in noise-damaged ears: a light and electron-microscopic study. In: Salvi R, Henderson D, Hamernik R, Colletti V (eds) Basic and applied aspects of noise-induced hearing loss. Plenum, New York, pp 163-176

Lilaonitkul W, Guinan JJ (2009) Reflex control of the human inner ear: a half-octave offset in medial efferent feedback that is consistent with an efferent role in the control of masking. J Neurophysiol 101:1394-1406

LoEHLIN JC (2004) Latent variable models: an introduction to factor, path, and structural equation analysis, 4th edn. Lawrence Erlbaum, Mahwah, pp 1-34

Lopez-Poveda EA, Alves-Pinto A (2008) A variant temporal masking curve method for inferring peripheral auditory compression. J Acoust Soc Am 123:1544-1554

Lopez-Poveda EA, Johannesen PT (2010) Towards designing a clinical method for estimating basilar response input/output response characteristics in listeners with normal and impaired hearing. Abstr Assoc Res Otolaryngol 33:47

Lopez-Poveda EA, Plack CJ, Meddis R (2003) Cochlear non-linearity between 500 and $8000 \mathrm{~Hz}$ in listeners with normal hearing. J Acoust Soc Am 113:951-960

Lopez-Poveda EA, Plack CJ, Meddis R (2005) Cochlear compression in listeners with moderate sensorineural hearing loss. Hear Res 205:172-183

Lopez-Poveda EA, Johannesen PT, Merchán MA (2009) Estimation of the degree of inner and outer hair cell dysfunction from distortion product otoacoustic emission input/output functions. Audiol Med 7:22-28

Maison SF, Liberman MC (2000) Predicting vulnerability to acoustic injury with a noninvasive assay of olivocochlear reflex strength. J Neurosci 20:4701-4707

Moore BCJ, Vickers DA, Plack CJ, Oxenham AJ (1999) Interrelationship between different psychoacoustic measures assumed to be related to the cochlear active mechanism. J Acoust Soc Am 106:2761-2778

Neely ST, Gorga MP, Dorn PA (2003) Cochlear compression estimates from measurements of distortion-product otoacoustic emissions. J Acoust Soc Am 114:1499-1507

Nelson DA, Schroder AC (1997) Linearized response growth inferred from growth-of-masking slopes in ears with cochlear hearing loss. J Acoust Soc Am 101:2186-2201

Nelson DA, Schroder AC, Wojtczak M (2001) A new procedure for measuring peripheral compression in normal-hearing and hearing-impaired listeners. J Acoust Soc Am 110:20452064 
Oxenham AJ, BACon SP (2003) Cochlear compression: perceptual measures and implications for normal and impaired hearing. Ear Hear 24:352-366

Oxenham AJ, Plack CJ (1997) A behavioral measure of basilarmembrane nonlinearity in listeners with normal and impaired hearing. J Acoust Soc Am 101:3666-3675

Oxenham AJ, Moore BCJ, Vickers DA (1997) Short-term temporal integration: evidence for the influence of peripheral compression. J Acoust Soc Am 101:3676-3687

Plack CJ, Skeels V (2007) Temporal integration and compression near absolute threshold in normal and impaired ears. J Acoust Soc Am 122:2236-2244

Plack CJ, Drga V, Lopez-Poveda EA (2004) Inferred basilar-membrane response function for listeners with mild to moderate sensorineural hearing loss. J Acoust Soc Am 115:1684-1695

Plack CJ, Oxenham AJ, Drga V (2006) Masking by inaudible sounds and the linearity of temporal summation. J Neurosci 26:87678773

Reed CM, Braida LD, Zurek PM (2009) Review of the literature on temporal resolution in listeners with cochlear hearing impairment: a critical assessment of the role of suprathreshold deficits. Trends Amplif 13:4-43

RHode WS, CoOper NP (1996) Nonlinear mechanics in the apical turn of the chinchilla cochlea in vivo. Aud Neurosci 3:101-121

Rhode WS, Recio A (2000) Study of mechanical motions in the basal region of the chinchilla cochlea. J Acoust Soc Am 107:33173332

Robles L, Ruggero MA (2001) Mechanics of the mammalian cochlea. Physiol Rev 81:1305-1352

Rosengard PS, Oxenham AJ, Braida LD (2005) Comparing different estimates of cochlear compression in listeners with normal and impaired hearing. J Acoust Soc Am 117:3028-3041

RugGero MA (1992) Responses to sound of the basilar membrane of the mammalian cochlea. Curr Opin Neurobiol 2:449-456

Ruggero MA, Rich NC, Recio A, NARAyAn SS, Robles L (1997) Basilarmembrane responses to tones at the base of the chinchilla cochlea. J Acoust Soc Am 101:2151-2163
Scheidt RE, Kale S, Heinz MG (2010) Noise-induced hearing loss alters the temporal dynamics of auditory-nerve responses. Hear Res 269:23-33

Schmiedt RA, Mills JM, Adams JC (1990) Tuning and suppression in auditory nerve fibers of aged gerbils raised in quiet or noise. Hear Res 45:221-236

Schmiedt RA, Lang H, Okamura H, Schulte BA (2002) Effects of furosemide applied chronically to the round window: a model of metabolic presbycusis. J Neurosci 22:9643-9650

Shaffer LA, Withnell RH, Dhar S, Lilly DJ, Goodman SS, Harmon KM (2003) Sources and mechanisms of DPOAE generation: implications for the prediction of auditory sensitivity. Ear Hear 24:367-379

Shera CA, Guinan JJ (1999) Evoked otoacoustic emissions arise by two fundamentally different mechanisms: a taxonomy for mammalian OAEs. J Acoust Soc Am 105:782-798

Sommers MS, GeHr SE (1998) Auditory suppression and frequency selectivity in older and younger adults. J Acoust Soc Am 103:1067-1074

STAinsBy TH, Moore BCJ (2006) Temporal masking curves for hearing-impaired listeners. Hear Res 218:98-111

Strouse A, Ashmead DH, Ohde RN, Grantham DW (1998) Temporal processing in the aging auditory system. J Acoust Soc Am 104:2385-2399

Williams EJ, BACON SP (2005) Compression estimates using behavioral and otoacoustic emission measures. Hear Res 201:44-54

Wojtczak M, Oxenham AJ (2009) Pitfalls in behavioral estimates of basilar-membrane compression in humans. J Acoust Soc Am 125:270-281

WOJTCZAK M, OXenham AJ (2010) Recovery from on- and offfrequency forward masking in listeners with normal and impaired hearing. J Acoust Soc Am 128:247-256

YASIN I, PLACK CJ (2003) The effects of a high-frequency suppressor on tuning curves and derived basilar-membrane response functions. J Acoust Soc Am 114:322-332

YATES GK (1990) Basilar membrane nonlinearity and its influence on auditory nerve rate-intensity functions. Hear Res 50:145-162 